\title{
Measurement of neutrino and antineutrino neutral-current quasielasticlike interactions on oxygen by detecting nuclear deexcitation $\gamma$ rays
}

K. Abe ${ }^{55}$ R. Akutsu ${ }^{56}$ A. Ali,${ }^{32}$ C. Alt, ${ }^{11}$ C. Andreopoulos, ${ }^{53,34}$ L. Anthony, ${ }^{34}$ M. Antonova, ${ }^{19}$ S. Aoki, ${ }^{31}$ A. Ariga,${ }^{2}$ Y. Ashida $\odot,{ }^{32}$ E. T. Atkin, ${ }^{21}$ Y. Awataguchi, ${ }^{58}$ S. Ban,${ }^{32}$ M. Barbi,${ }^{45}$ G. J. Barker, ${ }^{65}$ G. Barr, ${ }^{42}$ C. Barry, ${ }^{34}$

M. Batkiewicz-Kwasniak, ${ }^{15}$ A. Beloshapkin, ${ }^{26}$ F. Bench, ${ }^{34}$ V. Berardi, ${ }^{22}$ S. Berkman, ${ }^{4,61}$ L. Berns, ${ }^{57}$ S. Bhadra,${ }^{69}$ S. Bienstock, ${ }^{52}$ A. Blondel, ${ }^{13,}$ S. Bolognesi,${ }^{6}$ B. Bourguille, ${ }^{18}$ S. B. Boyd,${ }^{65}$ D. Brailsford, ${ }^{33}$ A. Bravar, ${ }^{13}$ D. Bravo Berguño, ${ }^{1}$ C. Bronner, ${ }^{55}$ A. Bubak,${ }^{50}$ M. Buizza Avanzini, ${ }^{10}$ J. Calcutt, ${ }^{36}$ T. Campbell, ${ }^{7}$ S. Cao, ${ }^{16}$ S. L. Cartwright ${ }^{49}$ M. G. Catanesi, ${ }^{22}$ A. Cervera, ${ }^{19}$ A. Chappell,${ }^{65}$ C. Checchia, ${ }^{24}$ D. Cherdack, ${ }^{17}$ N. Chikuma, ${ }^{54}$ G. Christodoulou, ${ }^{12}$ J. Coleman, ${ }^{34}$ G. Collazuol, ${ }^{24}$ L. Cook,${ }^{42,28}$ D. Coplowe,${ }^{42}$ A. Cudd ${ }^{36}$ A. Dabrowska, ${ }^{15}$ G. De Rosa, ${ }^{23}$ T. Dealtry ${ }^{33}$ P. F. Denner ${ }^{65}$ S. R. Dennis, ${ }^{34}$ C. Densham, ${ }^{53}$ F. Di Lodovico, ${ }^{30}$ N. Dokania ${ }^{39}$ S. Dolan, ${ }^{12}$ O. Drapier, ${ }^{10}$ J. Dumarchez ${ }^{52}$ P. Dunne, ${ }^{21}$ L. Eklund, ${ }^{14}$ S. Emery-Schrenk, ${ }^{6}$ A. Ereditato, ${ }^{2}$ P. Fernandez, ${ }^{19}$ T. Feusels, ${ }^{4,61}$ A. J. Finch, ${ }^{33}$ G. A. Fiorentini, ${ }^{69}$ G. Fiorillo, ${ }^{23}$ C. Francois, ${ }^{2}$ M. Friend,${ }^{16, \$}$ Y. Fujii, ${ }^{16,}$ R. Fujita, ${ }^{54}$ D. Fukuda, ${ }^{40}$ R. Fukuda,${ }^{59}$ Y. Fukuda,${ }^{37}$

K. Gameil, ${ }^{4,61}$ C. Giganti, ${ }^{52}$ T. Golan, ${ }^{67}$ M. Gonin, ${ }^{10}$ A. Gorin, ${ }^{26}$ M. Guigue, ${ }^{52}$ D. R. Hadley, ${ }^{65}$ J. T. Haigh, ${ }^{65}$

P. Hamacher-Baumann, ${ }^{48}$ M. Hartz, ${ }^{61,28}$ T. Hasegawa, ${ }^{16,}$ N. C. Hastings, ${ }^{16}$ T. Hayashino, ${ }^{32}$ Y. Hayato, ${ }^{55,28}$ A. Hiramoto, ${ }^{32}$ M. Hogan, ${ }^{8}$ J. Holeczek, ${ }^{50}$ N. T. Hong Van ${ }^{20,27}$ F. Iacob,${ }^{24}$ A. K. Ichikawa, ${ }^{32}$ M. Ikeda, ${ }^{55}$ T. Ishida, ${ }^{16, \$}$ T. Ishii, ${ }^{16, \sharp}$ M. Ishitsuka, ${ }^{59} \mathrm{~K}$. Iwamoto, ${ }^{54}$ A. Izmaylov, ${ }^{19,26}$ B. Jamieson, ${ }^{66}$ S. J. Jenkins ${ }^{49}$ C. Jesús-Valls, ${ }^{18} \mathrm{M}$. Jiang, ${ }^{32}$ S. Johnson, ${ }^{7}$ P. Jonsson, ${ }^{21}$ C. K. Jung, ${ }^{39,8}$ M. Kabirnezhad, ${ }^{42}$ A. C. Kaboth, ${ }^{47,53}$ T. Kajita ${ }^{56,8}$ H. Kakuno, ${ }^{58}$ J. Kameda,${ }^{55}$ D. Karlen, ${ }^{6,61}$ S. P. Kasetti, ${ }^{35}$ Y. Kataoka, ${ }^{55}$ T. Katori, ${ }^{30}$ Y. Kato, ${ }^{55}$ E. Kearns, ${ }^{3,28,8}$ M. Khabibullin, ${ }^{26}$ A. Khotjantsev, ${ }^{26}$ T. Kikawa, ${ }^{32}$ H. Kim, ${ }^{41}$ J. Kim, ${ }^{4,61}$ S. King, ${ }^{44}$ J. Kisiel,${ }^{50}$ A. Knight, ${ }^{65}$ A. Knox, ${ }^{33}$ T. Kobayashi, ${ }^{16,}$ L. Koch, ${ }^{53}$ T. Koga ${ }^{54}$ A. Konaka, ${ }^{61}$ L. L. Kormos, ${ }^{33}$ Y. Koshio, ${ }^{40,8}$ K. Kowalik, ${ }^{38}$ H. Kubo, ${ }^{32}$ Y. Kudenko, ${ }^{26, \|}$ N. Kukita, ${ }^{41}$ S. Kuribayashi, ${ }^{32}$ R. Kurjata, ${ }^{64}$ T. Kutter ${ }^{35}$ M. Kuze, ${ }^{57}$ L. Labarga, ${ }^{1}$ J. Lagoda,${ }^{38}$ M. Lamoureux, ${ }^{24}$ M. Laveder, ${ }^{24}$ M. Lawe, ${ }^{33}$ M. Licciardi, ${ }^{10}$ T. Lindner, ${ }^{61}$ R. P. Litchfield, ${ }^{14}$ S. L. Liu ${ }^{39}$ X. Li ${ }^{39}$ A. Longhin, ${ }^{24}$ L. Ludovici, ${ }^{25}$ X. Lu, ${ }^{42}$ T. Lux, ${ }^{18}$ L. N. Machado, ${ }^{23}$ L. Magaletti, ${ }^{22}$ K. Mahn ${ }^{36}$ M. Malek ${ }^{49}$ S. Manly, ${ }^{46}$ L. Maret ${ }^{13}$ A. D. Marino, ${ }^{7}$ J. F. Martin, ${ }^{60}$ T. Maruyama, ${ }^{16}$, T. Matsubara, ${ }^{16}$ K. Matsushita, ${ }^{54}$ V. Matveev ${ }^{26}$ K. Mavrokoridis ${ }^{34}$ E. Mazzucato, ${ }^{6}$ M. McCarthy ${ }^{69}$ N. McCauley,${ }^{34}$ K. S. McFarland, ${ }^{46}$ C. McGrew, ${ }^{39}$ A. Mefodiev ${ }^{26}$ C. Metelko, ${ }^{34}$ M. Mezzetto, ${ }^{24}$ A. Minamino, ${ }^{68}$ O. Mineev, ${ }^{26}$ S. Mine, ${ }^{5}$ M. Miura, ${ }^{55,8}$ L. Molina Bueno, ${ }^{11}$ S. Moriyama, ${ }^{55,8}$ J. Morrison, ${ }^{36}$ Th. A. Mueller, ${ }^{10}$ L. Munteanu, ${ }^{6}$ S. Murphy, ${ }^{11}$ Y. Nagai, ${ }^{7}$ T. Nakadaira, ${ }^{16, *}$ M. Nakahata, ${ }^{55,28}$ Y. Nakajima, ${ }^{55}$ A. Nakamura, ${ }^{40}$ K. G. Nakamura, ${ }^{32}$ K. Nakamura, ${ }^{28,16, "}$

S. Nakayama, ${ }^{55,28}$ T. Nakaya, ${ }^{32,28}$ K. Nakayoshi, ${ }^{16, \$}$ C. Nantais, ${ }^{60}$ T. V. Ngoc, ${ }^{20,9}$ K. Niewczas, ${ }^{67}$ K. Nishikawa, ${ }^{16, *}$ Y. Nishimura, ${ }^{29}$ T. S. Nonnenmacher, ${ }^{21}$ F. Nova, ${ }^{53}$ P. Novella, ${ }^{19}$ J. Nowak, ${ }^{33}$ J. C. Nugent, ${ }^{14}$ H. M. O'Keeffe, ${ }^{33}$

L. O’Sullivan, ${ }^{49}$ T. Odagawa, ${ }^{32}$ K. Okumura, ${ }^{56,28}$ T. Okusawa ${ }^{41}$ S. M. Oser, ${ }^{4,61}$ R. A. Owen, ${ }^{44}$ Y. Oyama ${ }^{16, *}$ V. Palladino, ${ }^{23}$ J. L. Palomino, ${ }^{39}$ V. Paolone, ${ }^{43}$ W. C. Parker,${ }^{47}$ P. Paudyal,${ }^{34}$ M. Pavin,${ }^{61}$ D. Payne, ${ }^{34}$ G. C. Penn,${ }^{34}$ L. Pickering ${ }^{36}$ C. Pidcott ${ }^{49}$ E. S. Pinzon Guerra, ${ }^{69}$ C. Pistillo, ${ }^{2}$ B. Popov,${ }^{52,{ }^{* *}}$ K. Porwit, ${ }^{50}$ M. Posiadala-Zezula, ${ }^{63}$ A. Pritchard ${ }^{34}$ B. Quilain, ${ }^{28}$ T. Radermacher, ${ }^{48}$ E. Radicioni, ${ }^{22}$ B. Radics, ${ }^{11}$ P. N. Ratoff, ${ }^{33}$ E. Reinherz-Aronis, ${ }^{8}$ C. Riccio, ${ }^{23}$ E. Rondio, ${ }^{38}$ S. Roth ${ }^{48}$ A. Rubbia, ${ }^{11}$ A. C. Ruggeri,${ }^{23}$ A. Rychter, ${ }^{64}$ K. Sakashita, ${ }^{16, \$}$ F. Sánchez,${ }^{13}$ C. M. Schloesser, ${ }^{11}$ K. Scholberg, ${ }^{9,8}$ J. Schwehr, ${ }^{8}$ M. Scott, ${ }^{21}$ Y. Seiya, ${ }^{41, \dagger}$ T. Sekiguchi, ${ }^{16, \$}$ H. Sekiya, ${ }^{55,28,8}$ D. Sgalaberna, ${ }^{12}$ R. Shah,${ }^{53,42}$ A. Shaikhiev, ${ }^{26}$ F. Shaker, ${ }^{66}$ A. Shaykina, ${ }^{26}$ M. Shiozawa, ${ }_{55}^{55,28}$ W. Shorrock, ${ }^{21}$ A. Shvartsman ${ }^{26}$ A. Smirnov, ${ }^{26}$ M. Smy, ${ }_{16}^{5}$ J. T. Sobczyk ${ }^{67}$ H. Sobel, ${ }^{5,28}$ F. J. P. Soler, ${ }^{14}$ Y. Sonoda, ${ }^{55}$ J. Steinmann, ${ }^{48}$ S. Suvorov, ${ }^{26,6}$ A. Suzuki, ${ }^{31}$ S. Y. Suzuki, ${ }^{16,}$ Y. Suzuki, ${ }^{28}$ A. A. Sztuc, ${ }^{21}$ M. Tada, ${ }^{16, \$}$ M. Tajima ${ }^{32}$ A. Takeda, ${ }^{55}$ Y. Takeuchi, ${ }^{31,28}$ H. K. Tanaka, ${ }^{55,}$ H. A. Tanaka, ${ }^{51,60}$ S. Tanaka, ${ }^{41}$ L. F. Thompson, ${ }^{49}$ W. Toki,${ }^{8}$ C. Touramanis,${ }^{34}$ K. M. Tsui, ${ }^{34}$ T. Tsukamoto ${ }^{16, \$}$ M. Tzanov, ${ }^{35}$ Y. Uchida, ${ }^{21}$ W. Uno ${ }^{32}$

M. Vagins, ${ }^{28,5}$ S. Valder, ${ }^{65}$ Z. Vallari ${ }^{39}$ D. Vargas, ${ }^{18}$ G. Vasseur, ${ }^{6}$ C. Vilela, ${ }^{39}$ W. G. S. Vinning, ${ }^{65}$ T. Vladisavljevic ${ }^{42,28}$ V. V. Volkov ${ }^{26}$ T. Wachala, ${ }^{15}$ J. Walker, ${ }^{66}$ J. G. Walsh, ${ }^{33}$ Y. Wang, ${ }^{39}$ D. Wark, ${ }^{53,42}$ M. O. Wascko, ${ }^{21}$ A. Weber, ${ }^{53,42}$ R. Wendell,,${ }^{32,8}$ M. J. Wilking, ${ }^{39}$ C. Wilkinson, ${ }^{2}$ J. R. Wilson, ${ }^{30}$ R. J. Wilson, ${ }^{8}$ K. Wood ${ }^{39}$ C. Wret, ${ }^{46}$ Y. Yamada, ${ }^{16,}$ K. Yamamoto, ${ }^{41, \dagger}$ C. Yanagisawa, ${ }^{39, \$ 1}$ G. Yang, ${ }^{39}$ T. Yano, ${ }^{55}$ K. Yasutome, ${ }^{32}$ S. Yen, ${ }^{61}$ N. Yershov ${ }^{26}$ M. Yokoyama, ${ }^{54,8}$ T. Yoshida ${ }^{57}$ M. Yu, ${ }^{69}$ A. Zalewska, ${ }^{15}$ J. Zalipska, ${ }^{38}$ K. Zaremba, ${ }^{64}$ G. Zarnecki, ${ }^{38}$ M. Ziembicki, ${ }^{64}$ E. D. Zimmerman, M. Zito, ${ }^{6}$ S. Zsoldos, ${ }^{44}$ and A. Zykova ${ }^{26}$

(T2K Collaboration)

${ }^{1}$ University Autonoma Madrid, Department of Theoretical Physics, Madrid, Spain

${ }^{2}$ University of Bern, Albert Einstein Center for Fundamental Physics,

Laboratory for High Energy Physics (LHEP), Bern, Switzerland

${ }^{3}$ Boston University, Department of Physics, Boston, Massachusetts, USA 
${ }^{4}$ University of British Columbia, Department of Physics and Astronomy, Vancouver, British Columbia, Canada

${ }^{5}$ University of California, Irvine, Department of Physics and Astronomy, Irvine, California, USA ${ }^{6}$ IRFU, CEA Saclay, Gif-sur-Yvette, France

${ }^{7}$ University of Colorado at Boulder, Department of Physics, Boulder, Colorado, USA

${ }^{8}$ Colorado State University, Department of Physics, Fort Collins, Colorado, USA

${ }^{9}$ Duke University, Department of Physics, Durham, North Carolina, USA

${ }^{10}$ Ecole Polytechnique, IN2P3-CNRS, Laboratoire Leprince-Ringuet, Palaiseau, France

${ }^{11}$ ETH Zurich, Institute for Particle Physics and Astrophysics, Zurich, Switzerland

${ }^{12}$ CERN European Organization for Nuclear Research, Genve 23, Switzerland

${ }^{13}$ University of Geneva, Section de Physique, DPNC, Geneva, Switzerland

${ }^{14}$ University of Glasgow, School of Physics and Astronomy, Glasgow, United Kingdom

${ }^{15}$ H. Niewodniczanski Institute of Nuclear Physics PAN, Cracow, Poland

${ }^{16}$ High Energy Accelerator Research Organization (KEK), Tsukuba, Ibaraki, Japan

${ }^{17}$ University of Houston, Department of Physics, Houston, Texas, USA

${ }^{18}$ Institut de Fisica d'Altes Energies (IFAE), The Barcelona Institute of Science and Technology, Campus UAB, Bellaterra (Barcelona) Spain

${ }^{19}$ IFIC (CSIC \& University of Valencia), Valencia, Spain

${ }^{20}$ Institute For Interdisciplinary Research in Science and Education (IFIRSE), ICISE, Quy Nhon, Vietnam

${ }^{21}$ Imperial College London, Department of Physics, London, United Kingdom

${ }^{22}$ INFN Sezione di Bari and Università e Politecnico di Bari,

Dipartimento Interuniversitario di Fisica, Bari, Italy

${ }^{23}$ INFN Sezione di Napoli and Università di Napoli, Dipartimento di Fisica, Napoli, Italy

${ }^{24}$ INFN Sezione di Padova and Università di Padova, Dipartimento di Fisica, Padova, Italy

${ }^{25}$ INFN Sezione di Roma and Università di Roma "La Sapienza", Roma, Italy

${ }^{26}$ Institute for Nuclear Research of the Russian Academy of Sciences, Moscow, Russia

${ }^{27}$ International Centre of Physics, Institute of Physics (IOP), Vietnam Academy of Science and Technology (VAST), 10 Dao Tan, Ba Dinh, Hanoi, Vietnam

${ }^{28}$ Kavli Institute for the Physics and Mathematics of the Universe (WPI), The University of Tokyo Institutes

for Advanced Study, University of Tokyo, Kashiwa, Chiba, Japan

${ }^{29}$ Keio University, Department of Physics, Kanagawa, Japan

${ }^{30}$ King's College London, Department of Physics, Strand, London, United Kingdom

${ }^{31}$ Kobe University, Kobe, Japan

${ }^{32}$ Kyoto University, Department of Physics, Kyoto, Japan

${ }^{33}$ Lancaster University, Physics Department, Lancaster, United Kingdom

${ }^{34}$ University of Liverpool, Department of Physics, Liverpool, United Kingdom

${ }^{35}$ Louisiana State University, Department of Physics and Astronomy, Baton Rouge, Louisiana, USA

${ }^{36}$ Michigan State University, Department of Physics and Astronomy, East Lansing, Michigan, USA

${ }^{37}$ Miyagi University of Education, Department of Physics, Sendai, Japan

${ }^{38}$ National Centre for Nuclear Research, Warsaw, Poland

${ }^{39}$ State University of New York at Stony Brook, Department of Physics and Astronomy,

Stony Brook, New York, USA

${ }^{40}$ Okayama University, Department of Physics, Okayama, Japan

${ }^{41}$ Osaka City University, Department of Physics, Osaka, Japan

${ }^{42}$ Oxford University, Department of Physics, Oxford, United Kingdom

${ }^{43}$ University of Pittsburgh, Department of Physics and Astronomy, Pittsburgh, Pennsylvania, USA

${ }^{44}$ Queen Mary University of London, School of Physics and Astronomy, London, United Kingdom

${ }^{45}$ University of Regina, Department of Physics, Regina, Saskatchewan, Canada

${ }^{46}$ University of Rochester, Department of Physics and Astronomy, Rochester, New York, USA

${ }^{47}$ Royal Holloway University of London, Department of Physics, Egham, Surrey, United Kingdom

${ }^{48}$ RWTH Aachen University, III. Physikalisches Institut, Aachen, Germany

${ }^{49}$ University of Sheffield, Department of Physics and Astronomy, Sheffield, United Kingdom

${ }^{50}$ University of Silesia, Institute of Physics, Katowice, Poland

${ }^{51}$ SLAC National Accelerator Laboratory, Stanford University, Menlo Park, California, USA

${ }^{52}$ Sorbonne Université, Université Paris Diderot, CNRS/IN2P3, Laboratoire de Physique Nucléaire et de Hautes Energies (LPNHE), Paris, France

${ }^{53}$ STFC, Rutherford Appleton Laboratory, Harwell Oxford, and Daresbury Laboratory,

Warrington, United Kingdom

${ }^{54}$ University of Tokyo, Department of Physics, Tokyo, Japan

${ }^{55}$ University of Tokyo, Institute for Cosmic Ray Research, Kamioka Observatory, Kamioka, Japan 


\author{
${ }^{56}$ University of Tokyo, Institute for Cosmic Ray Research, \\ Research Center for Cosmic Neutrinos, Kashiwa, Japan \\ ${ }^{57}$ Tokyo Institute of Technology, Department of Physics, Tokyo, Japan \\ ${ }^{58}$ Tokyo Metropolitan University, Department of Physics, Tokyo, Japan \\ ${ }^{59}$ Tokyo University of Science, Faculty of Science and Technology, \\ Department of Physics, Noda, Chiba, Japan \\ ${ }^{60}$ University of Toronto, Department of Physics, Toronto, Ontario, Canada \\ ${ }^{61}$ TRIUMF, Vancouver, British Columbia, Canada \\ ${ }^{62}$ University of Victoria, Department of Physics and Astronomy, Victoria, British Columbia, Canada \\ ${ }^{63}$ University of Warsaw, Faculty of Physics, Warsaw, Poland \\ ${ }^{64}$ Warsaw University of Technology, Institute of Radioelectronics and Multimedia Technology, \\ Warsaw, Poland \\ ${ }^{65}$ University of Warwick, Department of Physics, Coventry, United Kingdom \\ ${ }^{66}$ University of Winnipeg, Department of Physics, Winnipeg, Manitoba, Canada \\ ${ }^{67}$ Wroclaw University, Faculty of Physics and Astronomy, Wroclaw, Poland \\ ${ }^{68}$ Yokohama National University, Faculty of Engineering, Yokohama, Japan \\ ${ }^{69}$ York University, Department of Physics and Astronomy, Toronto, Ontario, Canada
}

(Received 21 October 2019; published 30 December 2019)

\begin{abstract}
Neutrino- and antineutrino-oxygen neutral-current quasielasticlike interactions are measured at SuperKamiokande using nuclear deexcitation $\gamma$ rays to identify signal-like interactions in data from a 14.94(16.35) $\times$ $10^{20}$ protons-on-target exposure of the T2K neutrino (antineutrino) beam. The measured flux-averaged cross sections on oxygen nuclei are $\left\langle\sigma_{\nu \text {-NCQE }}\right\rangle=1.70 \pm 0.17$ (stat. $)_{-0.38}^{+0.51}$ (syst. $) \times 10^{-38} \mathrm{~cm}^{2} /$ oxygen with a fluxaveraged energy of $0.82 \mathrm{GeV}$ and $\left\langle\sigma_{\bar{\nu}-\mathrm{NCQE}}\right\rangle=0.98 \pm 0.16$ (stat. $)_{-0.19}^{+0.26}$ (syst.) $\times 10^{-38} \mathrm{~cm}^{2} /$ oxygen with a flux-averaged energy of $0.68 \mathrm{GeV}$, for neutrinos and antineutrinos, respectively. These results are the most precise to date, and the antineutrino result is the first cross section measurement of this channel. They are compared with various theoretical predictions. The impact on evaluation of backgrounds to searches for supernova relic neutrinos at present and future water Cherenkov detectors is also discussed.
\end{abstract}

DOI: 10.1103/PhysRevD.100.112009

\section{INTRODUCTION}

Measurements of neutrino neutral-current $(\mathrm{NC})$ processes give insight into neutrino-nucleus interactions and are important for understanding the nucleon itself as well as improving the sensitivity of searches for a variety of physics

\footnotetext{
${ }^{*}$ Deceased.

†Present address: CERN

${ }^{*}$ Also at J-PARC, Tokai, Japan.

${ }^{\S}$ Affiliated member at Kavli IPMU (WPI), the University of Tokyo, Japan.

"Also at National Research Nuclear University "MEPhI" and Moscow Institute of Physics and Technology, Moscow, Russia.

"Also at the Graduate University of Science and Technology, Vietnam Academy of Science and Technology.

**Also at JINR, Dubna, Russia.

Also at Nambu Yoichiro Institute of Theoretical and Experimental Physics (NITEP).

\#Also at BMCC/CUNY, Science Department, New York, New York, USA.

Published by the American Physical Society under the terms of the Creative Commons Attribution 4.0 International license. Further distribution of this work must maintain attribution to the author(s) and the published article's title, journal citation, and DOI. Funded by SCOAP ${ }^{3}$.
}

phenomena. The strange quark content of the nucleon $(\Delta s)$, for instance, can be probed via NC interactions (see Ref. [1] and references therein), and its measurements have been demonstrated by the BNL E734 experiment [2] and the MiniBooNE experiment $[3,4]$. Precision measurements of the neutrino- and antineutrino-oxygen NC interactions in the sub-GeV region, where the quasielastic process is expected to be dominant, also benefit a diverse array of searches with water Cherenkov detectors, such as Super-Kamiokande (SK) [5], its future upgrade, SK-Gd [6], and its successor, Hyper-Kamiokande [7]. In supernova relic neutrino (SRN) searches [8-10], the present uncertainty on these interactions induces a large error on atmospheric neutrino backgrounds, limiting the sensitivity at low energies where the SRN flux is predicted to be large. When searching for dark matter in accelerator neutrino experiments, as suggested in Refs. [11,12], the rate of NC interactions must be accurately estimated as they are indistinguishable from the signal. Another motivation arises in the search for sterile neutrinos in accelerator neutrino experiments [13-15]. The fact that the NC interaction cross section does not depend on the neutrino flavor makes it possible to search for a deficit of $\mathrm{NC}$ events, which would be interpreted as transitions from active to sterile neutrinos. 
$\mathrm{NC}$ interactions at the neutrino energies of interest here $\left(E_{\nu} \lesssim 1 \mathrm{GeV}\right)$ are difficult to observe in water Cherenkov detectors because their final state particles are either neutral or charged but often below the Cherenkov threshold. Instead, the present work seeks to identify these interactions using Cherenkov light arising from the electromagnetic cascade produced by $\gamma$ rays emitted from the deexcitation of the recoil nucleus [16-19]. At $E_{\nu} \gtrsim 200 \mathrm{MeV}$, the NC quasielastic nucleon knock-out (NCQE) processes,

$$
\begin{aligned}
& \nu(\bar{\nu})+{ }^{16} \mathrm{O} \rightarrow \nu(\bar{\nu})+n+{ }^{15} \mathrm{O}^{*}, \\
& \nu(\bar{\nu})+{ }^{16} \mathrm{O} \rightarrow \nu(\bar{\nu})+p+{ }^{15} \mathrm{~N}^{*},
\end{aligned}
$$

become dominant over $\mathrm{NC}$ inelastic processes without nucleon knock-out, $\nu(\bar{\nu})+{ }^{16} \mathrm{O} \rightarrow \nu(\bar{\nu})+{ }^{16} \mathrm{O}^{*}$ [19]. The resulting excited nuclei relax to the ground state with the emission of $\gamma$ rays promptly. These $\gamma$ rays are available as a probe to study the NCQE interaction as has been demonstrated at T2K [20] and SK [21]. Previous studies at $\mathrm{T} 2 \mathrm{~K}$ measured the neutrino-oxygen NCQE interaction cross section with a data set of $3.01 \times 10^{20}$ protons-ontarget (POT) and SK measured this process with its atmospheric neutrino data, which is a mixture of neutrino and antineutrino interactions. Both measurements suffer from large statistical and systematic uncertainties.

This paper reports the updated result from T2K using neutrinos and the first measurement using antineutrinos. In this work the signal is termed "NCQE-like," to highlight the fact that the event selection may contain contributions from $\mathrm{NC}$ two-particle-two-hole $(2 \mathrm{p} 2 \mathrm{~h})$ interactions where two nucleons are involved in the interaction via mesonexchange currents. Previous studies $[20,21]$ may have also included such events, though they were not addressed specifically. Further descriptions will be given in Sec. VII. In the analysis, data taken with exposures of $14.94 \times 10^{20}$ POT in neutrino mode and $16.35 \times 10^{20}$ POT in antineutrino mode are used. Both the statistical and systematic errors have been reduced with the present analysis.

The paper is structured as follows. First, Sec. II details the experimental setup of T2K. Section III explains the Monte Carlo (MC) simulation and is followed by descriptions of the event reconstruction and selection in Sec. IV. Estimates of uncertainties in the analysis are described in Sec. V before cross section results are given in Sec. VI. After discussion of the results in Sec. VII concluding remarks are given in Sec. VIII.

\section{THE T2K EXPERIMENT}

The T2K experiment [22] has been designed for precise measurement of neutrino oscillation parameters [23] and has a broad program of additional physics measurements. It consists of the J-PARC neutrino beamline, near detectors, and SK as its far detector. T2K has taken data in nine separate run periods, termed Runs 1-9, and its beam intensity has increased throughout. Protons are bundled into eight bunches (six in Run 1), referred to as a spill, and accelerated to $30 \mathrm{GeV} / \mathrm{c}$ by the J-PARC Main Ring synchrotron. Bunches are approximately $100 \mathrm{~ns}$ wide and separated by about $580 \mathrm{~ns}$ and spills are delivered to the neutrino production target with a repetition rate of 2.48 s. Hadrons produced in proton-target (graphite) interactions are efficiently focused and sign-selected by magnetic fields produced by three electromagnetic horns $[24,25]$, before entering a decay volume. The polarity of the magnetic horns can be changed, allowing selection and focusing of either positively or negatively charged hadrons to produce beams composed of predominantly neutrinos or antineutrinos following the decay of the hadrons. The former is referred to as forward horn current (FHC) mode while the latter is referred to as reverse horn current (RHC) mode. Located $280 \mathrm{~m}$ away from the graphite target the two near detectors, INGRID [26] and ND280 [27,28], are placed on-axis and $2.5^{\circ}$ off-axis with respect to the proton beam direction, respectively. ND280 is used to measure the (anti)neutrino spectrum before the onset of neutrino oscillations and INGRID monitors the (anti)neutrino beam direction and intensity to ensure beam quality during data taking. In addition to the INGRID measurements a muon monitor placed just after the decay volume measures the beam direction and intensity on a bunch-by-bunch basis by detecting muons from pion and kaon decays [29-31].

Super-Kamiokande is located $295 \mathrm{~km}$ away from the target and $2.5^{\circ}$ off-axis. Beam timing information is shared between J-PARC and SK via a GPS system. It is a cylindrical water Cherenkov detector located 1,000 m under Mt. Ikeno in Kamioka, Japan. The detector is divided into two parts, an inner detector (ID) and an outer detector (OD). The ID measures $33.8 \mathrm{~m}$ in diameter and $36.2 \mathrm{~m}$ in height and is instrumented with 11,129 20-inch inwardfacing photomultiplier tubes (PMTs) on its wall, while the entire detector volume, which includes the $\sim 2 \mathrm{~m}$ thick OD region, extends $2.75 \mathrm{~m}$ radially and $2.6 \mathrm{~m}$ above and below the ID. Serving primarily as a veto, the OD is equipped with 1,885 8-inch outward-facing PMTs attached on the back side of the ID wall. The entire volume is filled with $50 \mathrm{kton}$ of ultra-pure water. In the present work, data from the fourth stage of the detector, known as SK-IV, are used. Further descriptions of SK can be found in Ref. [5].

\section{EVENT SIMULATION}

Simulation of the signal and background processes are essential to the optimization of the event selection and determination of systematic uncertainties in this analysis. Monte Carlo (MC) events generated according to models of neutrino beam, neutrino interactions, and the detector response including the $\gamma$ ray emission are considered. 


\section{A. Neutrino flux}

The neutrino flux is estii th mated by simulation based on FLUKA2011 [32] and GEANT3 [33] for modeling hadronic interactions and particle transport and decays in the beamline. Pion and kaon production cross sections are renormalized using data from the NA61/SHINE experiment taken using both thin and T2K replica targets [34-38]. Oscillations are taken into account for neutrinos that produce charged-current (CC) interactions at SK, using parameters from the recent $\mathrm{T} 2 \mathrm{~K}$ measurements [23]. Figure 1 shows the predicted $\mathrm{T} 2 \mathrm{~K}$ fluxes in the FHC and RHC modes without neutrino oscillations.

\section{B. Neutrino interaction}

NEUT (version 5.3.3) [39] is used to simulate neutrinonucleon interactions and subsequent final state interactions inside the target nucleus. For NCQE interactions the nominal nucleon momentum distribution is based on the Benhar spectral function $[19,40]$, while for CC quasielastic (CCQE) interactions the relativistic Fermi gas model [41] is used. The axial-vector mass is $M_{\mathrm{A}}^{\mathrm{QE}}=1.21 \mathrm{GeV} / \mathrm{c}^{2}$ and the Fermi
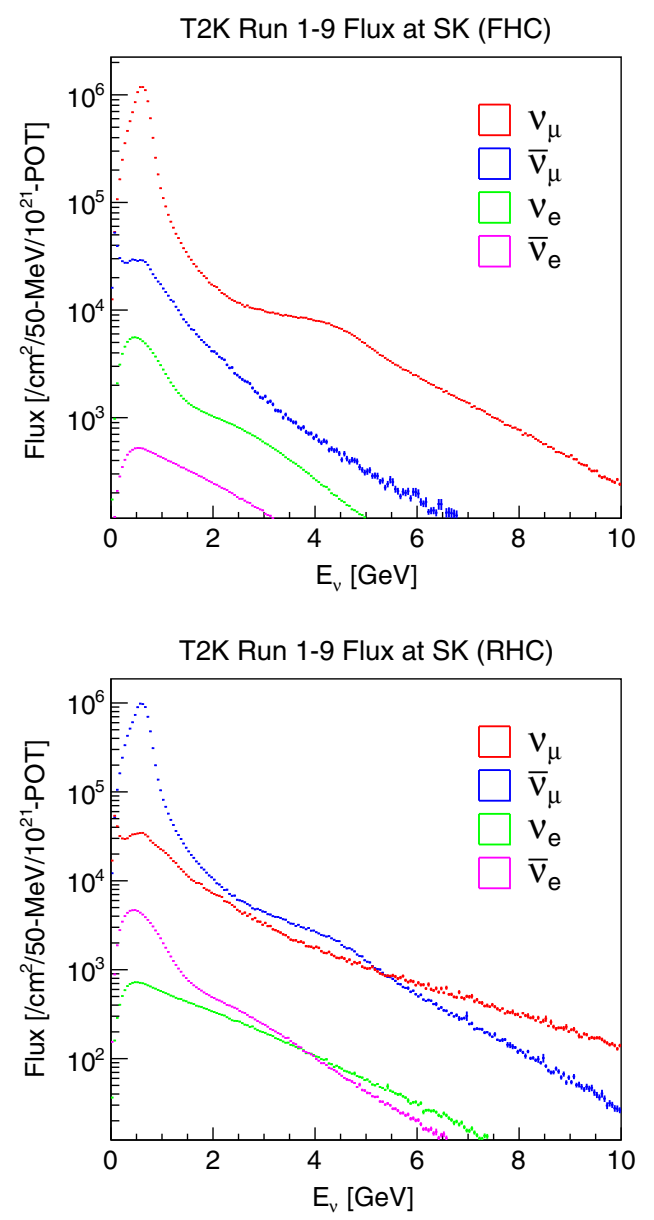

FIG. 1. T2K neutrino flux predictions at SK for the FHC (top) and RHC (bottom) operation modes without neutrino oscillations. momentum for oxygen is $225 \mathrm{MeV} / \mathrm{c}$. CC 2p2h interactions are modeled with the calculation in Ref. [42], but their neutral counterpart is not implemented in NEUT since no model is available in the literature. The simulation uses BBBA05 vector form factors [43] and a dipole axial-vector form factor. Single pion production is based on the model of Rein and Sehgal [44]. The axial-vector mass in the resonance interaction is $M_{\mathrm{A}}^{\mathrm{RES}}=0.95 \mathrm{GeV} / \mathrm{c}^{2}$. Deep inelastic scattering is simulated using the GRV98 parton distribution [45] with corrections by Bodek and Yang [46]. The final state interactions of hadrons inside the nucleus are simulated with a cascade model as described in Refs. [39,47]. Further simulation details are given in Ref. [47].

\section{C. $\gamma$ ray emission and detector response}

The emission of $\gamma$ rays from nuclear de-excitation is a key part of this analysis and is simulated separately for those produced by the neutrino-nucleus interactions (primary- $\gamma$ ) and those from nucleon-nucleus interactions (secondary- $\gamma$ ). These processes are schematically illustrated in Fig. 2.

After the initial neutrino interaction an excited state of the remaining nucleus is selected based on the probabilities calculated in Ref. [19]. There are four possible states, $\left(p_{1 / 2}\right)^{-1},\left(p_{3 / 2}\right)^{-1},\left(s_{1 / 2}\right)^{-1}$, and others. Here $(\text { state })^{-1}$ represents the state of the nucleus after a nucleon that initially occupied states $=\mathrm{p}_{1 / 2}, \mathrm{p}_{3 / 2}, \mathrm{~s}_{1 / 2}$ is removed from the nucleus. The probability for each of four states to be produced is $0.158,0.3515,0.1055$, and 0.385 , respectively [19]. The $\left(\mathrm{p}_{1 / 2}\right)^{-1}$ state is the ground state of ${ }^{15} \mathrm{O}$ or ${ }^{15} \mathrm{~N}$ and therefore leads to no $\gamma$ ray emission. Conversely, $\left(p_{3 / 2}\right)^{-1}$ almost always emits one $\gamma$ ray, with $6.18 \mathrm{MeV}$ from ${ }^{15} \mathrm{O}$ and $6.32 \mathrm{MeV}$ from ${ }^{15} \mathrm{~N}$ being the most likely. Since $\left(\mathrm{s}_{1 / 2}\right)^{-1}$ is a higher excited state, the branching fraction to decays including nucleons or alpha particles may be large. After such decays, the resulting nuclei may decay with $\gamma$ ray emission if it is still in an excited state thereafter. The others state includes all other possibilities and mainly includes contributions from short-range correlations among

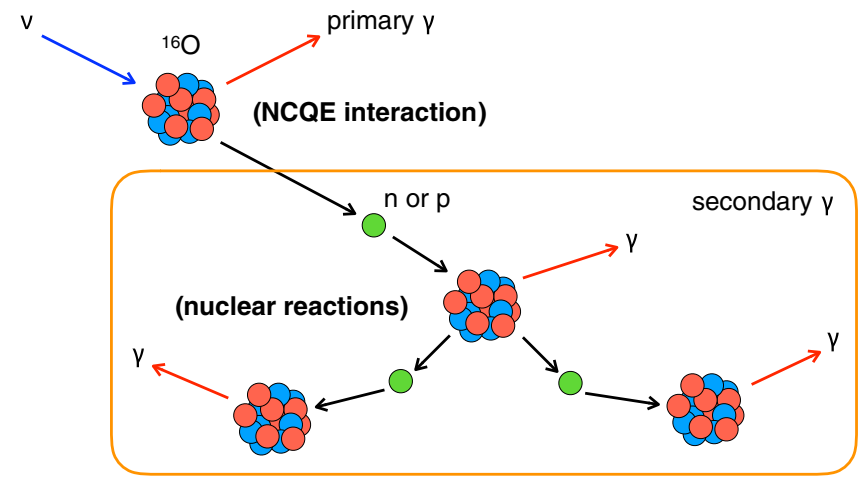

FIG. 2. Schematic of primary and secondary $\gamma$ rays in the NCQE interaction. 
nucleons. At present there is no data nor theoretical predictions of $\gamma$ ray emission for the states covered by others so in the nominal simulation they are integrated into $\left(\mathrm{s}_{1 / 2}\right)^{-1}$. A systematic uncertainty stemming from this choice is described in Sec. V. Further detailed descriptions on the treatment of these states are given in Ref. [20].
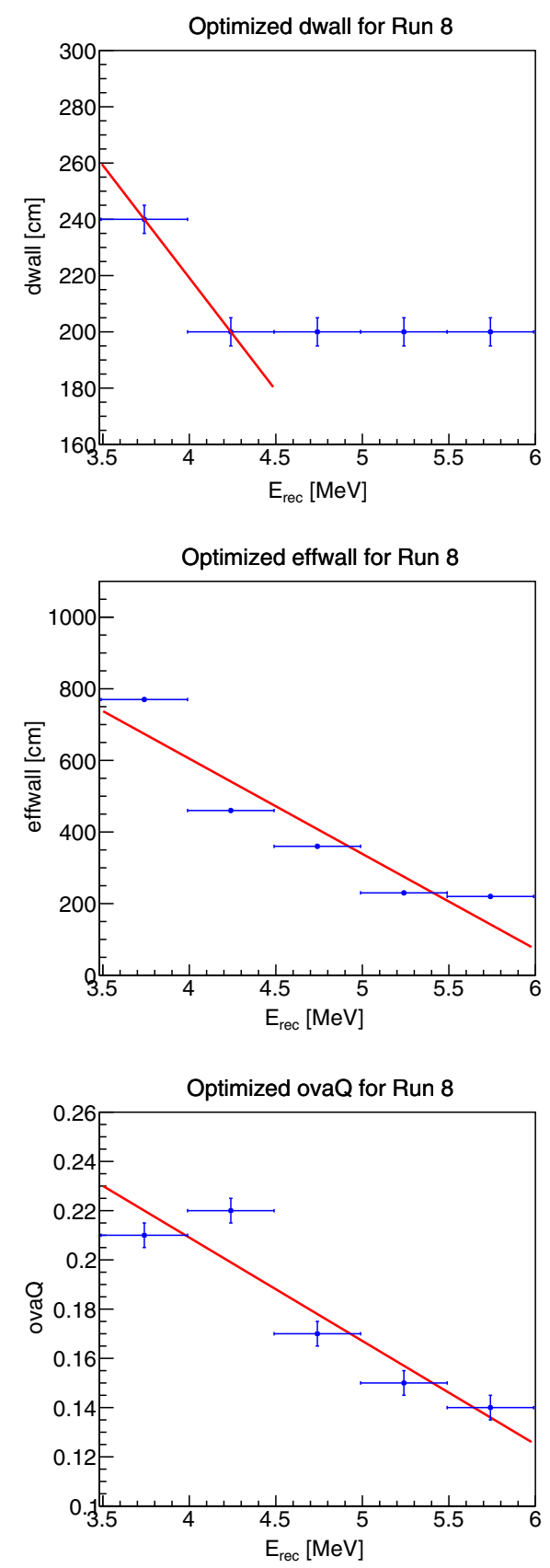

FIG. 3. Optimized cut values for $d$ wall (top), effwall (middle), and ovaQ (bottom), in each low energy bin for one of the FHC mode runs (Run 8). Vertical bars on each point represent the bin width used in parameter scans. Red lines represent linear fits to the distributions and are used for the cut values. Events with parameter values above the lines are used in the analysis. The fit regions for $d$ wall and effwall are explained in the text.
The interactions of secondary particles inside SK and the response of its PMTs are simulated with a GEANT3based package [33]. Hadronic interactions are of particular importance to the present analysis, especially models of neutron-nucleus reactions and the resulting $\gamma$ ray emission. These are handled by GCALOR $[48,49]$, which implements the MICAP model for neutrons below $20 \mathrm{MeV}$ and NMTC above $20 \mathrm{MeV}$. The MICAP model uses experimental cross sections from the ENDF/B-V library [50], while NMTC is based on an intranuclear cascade model.

\section{RECONSTRUCTION AND SELECTION}

Each event in SK is reconstructed with tools used for solar neutrino analysis [51-53]. The visible energy $\left(E_{\text {rec }}\right)$ is reconstructed using the number of hit PMTs. At these energies PMTs usually have registered only one
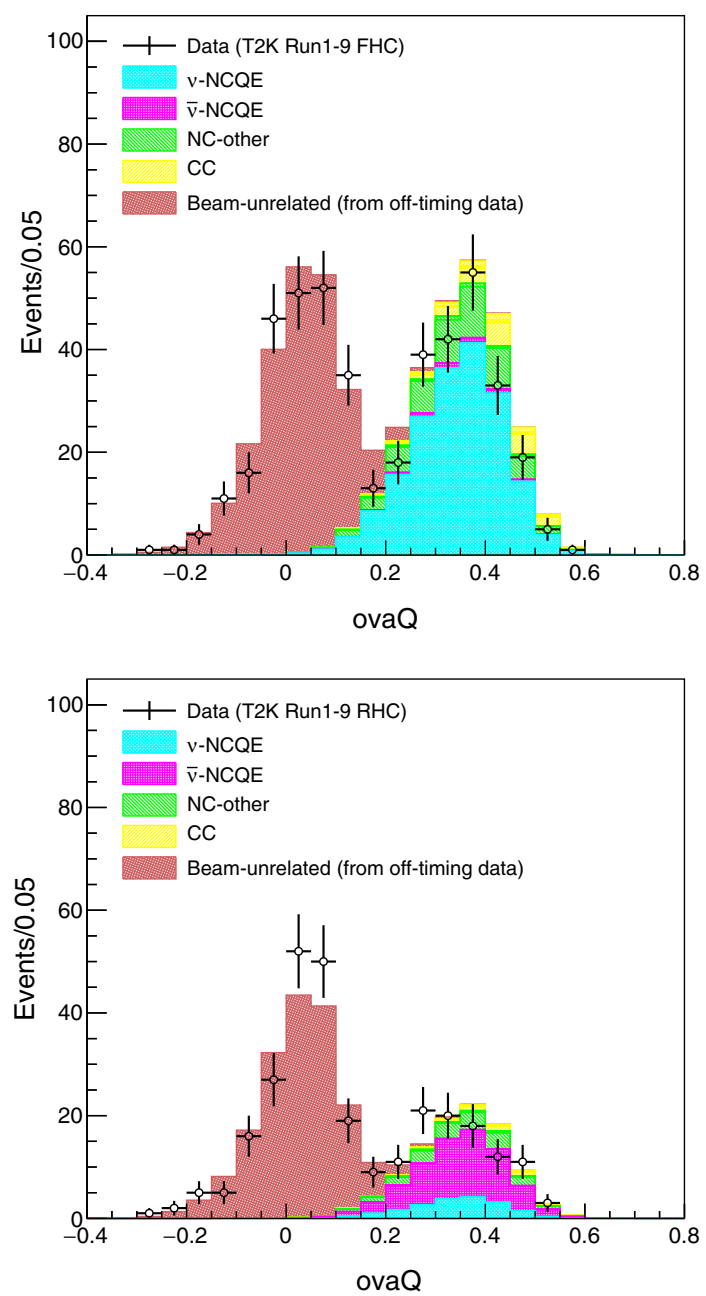

FIG. 4. Distributions of $o v a Q$ for FHC (top) and RHC (bottom) after the cuts in (1), the FV cut, the dwall cut, and the effwall cut. The MC prediction is broken down into four interactions: neutrino and antineutrino NCQE, NC-other, and CC. Beamunrelated events are obtained from the off-timing data as explained in the text. 

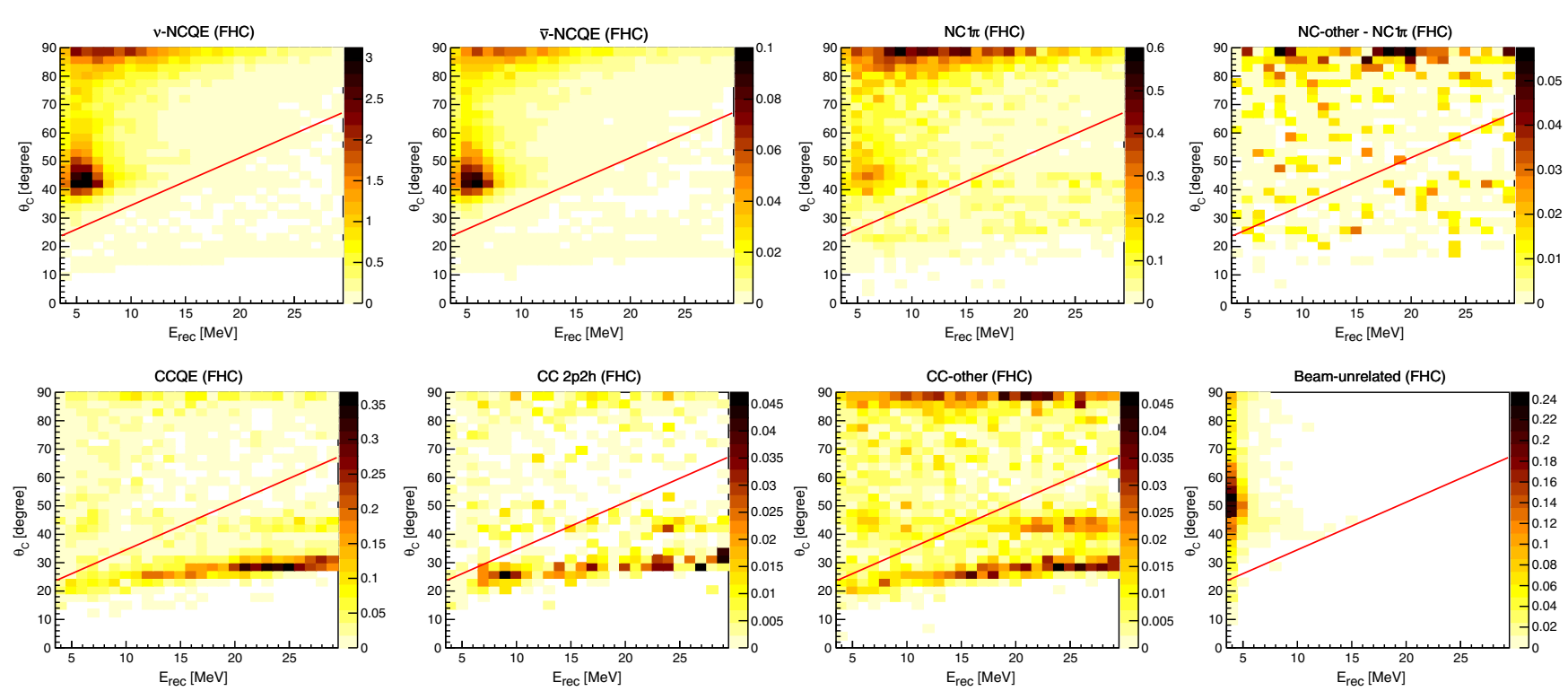

FIG. 5. Two-dimensional $E_{\mathrm{rec}}-\theta_{\mathrm{C}}$ distributions of each neutrino interaction channel by MC and beam-unrelated events by the offtiming data in FHC mode; the optimized linear function for the CC interaction cut is shown in red. Events above the line are used in the analysis. The $\mathrm{z}$-axis represents the predicted number of events [/MeV/2.7-degree] in the T2K Run 1-9 FHC mode. NC1 $\pi$ represents neutrino and antineutrino neutral-current interactions with a pion production, and $\mathrm{CC}$-other represents all other $\mathrm{CC}$ interactions than CCQE and CC 2p2h.

photoelectron and there are typically between 10 and 200 hit PMTs in the current analysis window. Note that the definition of energy in the present work differs from the previous T2K work [20], where the electron mass $(0.511 \mathrm{MeV})$ was added to the visible energy. The current definition is consistent with recent low energy analyses in SK [21,53]. The interaction vertex and direction are inferred from the PMT hit pattern and timing. A Cherenkov angle $\left(\theta_{\mathrm{C}}\right)$ for each event is calculated as the most frequently occurring value in the distribution of opening angles to all three-hit combinations of PMTs. Various calibrations are used to evaluate the performance of the reconstruction as detailed in Refs. [54,55].

This analysis considers five event categories, neutrino NCQE interactions (" $\nu$-NCQE"), antineutrino NCQE interactions (" $\bar{\nu}$-NCQE"), all other $\mathrm{NC}$ interactions ("NCother"), CC interactions, and accidental (beam-unrelated) backgrounds. Both the NC-other and CC categories include contributions from neutrinos and antineutrinos. Note that these event categories reflect the neutrino interaction prior to additional particle interactions within the nucleus. This means that, for example, the NC-other sample contains pion production events where a pion was produced but was later absorbed in the nucleus. The first four interactions are simulated using NEUT and beam-unrelated backgrounds are estimated using data outside of the T2K spill timing window. Event selection criteria are tuned to effectively select signal events, $\nu$-NCQE and $\bar{\nu}$-NCQE interactions, while removing other events as follows.

(1) Events are required to be in the energy range 3.49 to 29.49 MeV, above which CC interactions become dominant. Only data judged to be of good quality, based on the beam and detector conditions during each spill, are used [47]. To select beam-induced events with high purity, the reconstructed event timing is required to be within $\pm 100 \mathrm{~ns}$ of the expected timing of each bunch ("on-timing"). A sample of beam-unrelated events is selected by applying the same energy and quality cuts in a time window $[-500,-5] \mu$ s before the beam spill ("offtiming"). Events with hit clusters in a window spanning 20 to $0.2 \mu$ s before the event trigger which are consistent with activity from electrons produced in the muon or pion decay chain (decay-e's) are removed. The effect on the signal efficiency by this cut is negligible.

(2) Several additional event selection cuts are applied to remove backgrounds from radioactive impurities from the detector walls. First, a fiducial volume (FV) cut is applied to all events, which requires the distance between the reconstructed vertex position and the ID wall (dwall) to be more than $200 \mathrm{~cm}$. Below $6 \mathrm{MeV}$ radioactive backgrounds increase considerably, requiring tighter $d$ wall and reconstructed event quality cuts. Cuts in this energy region are tuned (discussed below) using three variables, dwall, effwall, and ovaQ. Here effwall is the distance from the event vertex to the ID wall as measured backward along the reconstructed track direction. The ovaQ parameter is a measure of the reconstruction quality and is defined as the difference of two parameters, ova $Q=g_{\mathrm{vtx}}^{2}-g_{\mathrm{dir}}^{2}$, 

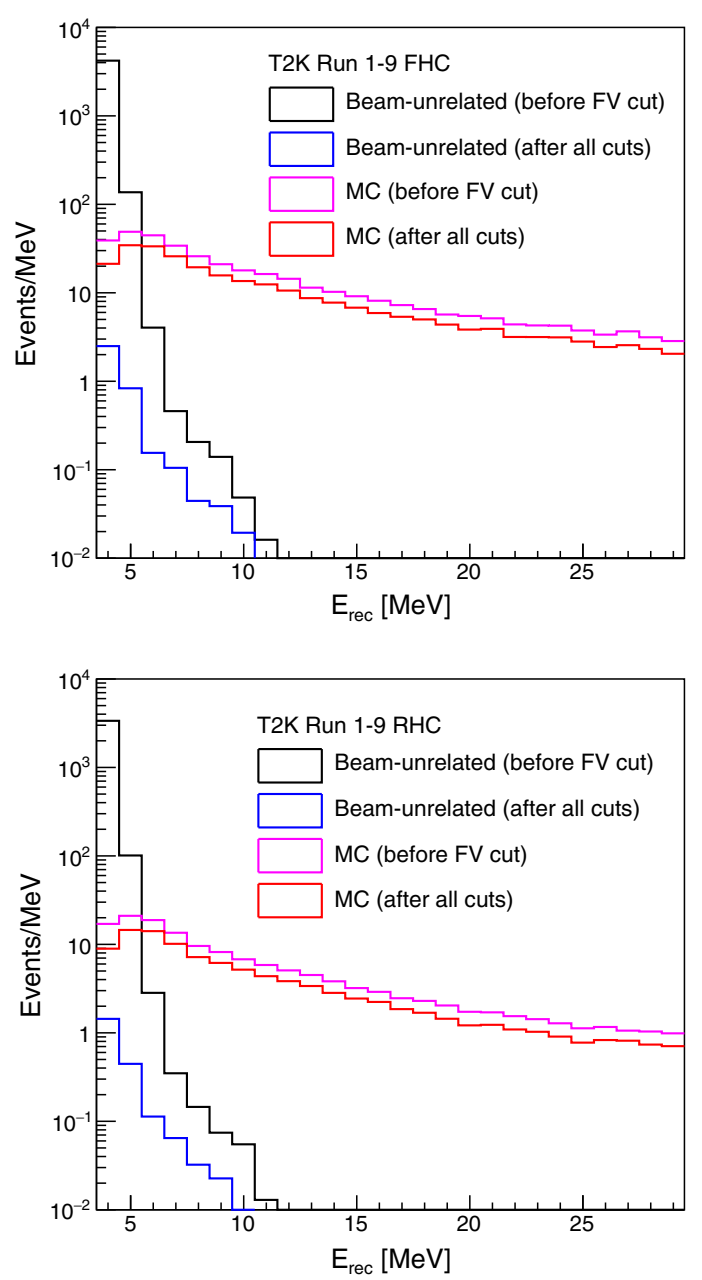

FIG. 6. Reconstructed energy distributions of MC and beamunrelated events before the FV cut and after all cuts for FHC (top) and RHC (bottom).

where $g_{\mathrm{vtx}}$ and $g_{\mathrm{dir}}$ are the vertex and direction fit quality parameters, respectively [56]. Cuts on these parameters are optimized for five regions between 3.49 and $5.99 \mathrm{MeV}$ with each $0.5 \mathrm{MeV}$ bin width.
The optimization is performed separately for each $\mathrm{T} 2 \mathrm{~K}$ run period because the detector condition and the beam power differ from run to run. A figure-ofmerit (FOM) designed to maximize sensitivity to the NCQE signal is defined as:

$$
\mathrm{FOM}=\frac{N_{\text {sig }}}{\sqrt{N_{\text {sig }}+N_{\text {bkg }}}},
$$

where $N_{\text {sig }}$ is the number of signal events predicted by the MC $(\nu$-NCQE for FHC and $\bar{\nu}$-NCQE for RHC) and $N_{\mathrm{bkg}}$ is the total number of background events. The latter is composed of two components, $N_{\text {bkg }}^{\mathrm{MC}}$ and $N_{\text {bkg }}^{\text {beam-unrelated }}$, which represent nonsignal neutrino events such as $\mathrm{NC}$-other and $\mathrm{CC}$ interactions, and beam-unrelated events from the offtiming data sample, respectively. Cuts on the three parameters above are chosen to maximize the FOM in each energy region. As an illustration the optimized values of $d$ wall, effwall, and ovaQ for one of the FHC mode runs (T2K Run 8) are shown in Fig. 3. A linear function is fit to each distribution to obtain the final cut criteria and is denoted by the red line in the figure. For the dwall and effwall distributions, if the optimized value is $200 \mathrm{~cm}$ (the FV cut criterion) in two successive energy bins, the second and later bins are removed and the fit is repeated. In the end, each of these three parameters is required to be larger than the obtained line. That is, events with values in the upper right portion of the plots in the figure are kept. Note that at higher energies the optimum dwall and effwall values fall below $200 \mathrm{~cm}$, but such events are already removed by the initial FV cut. Figure 4 shows the ovaQ distributions after the cuts described in (1), the FV cut, the optimized $d$ wall cut, and the optmized effwall cut. There is clear separation between signal and background. Further descriptions of the variables used in this selection are given in Refs. [20,56].
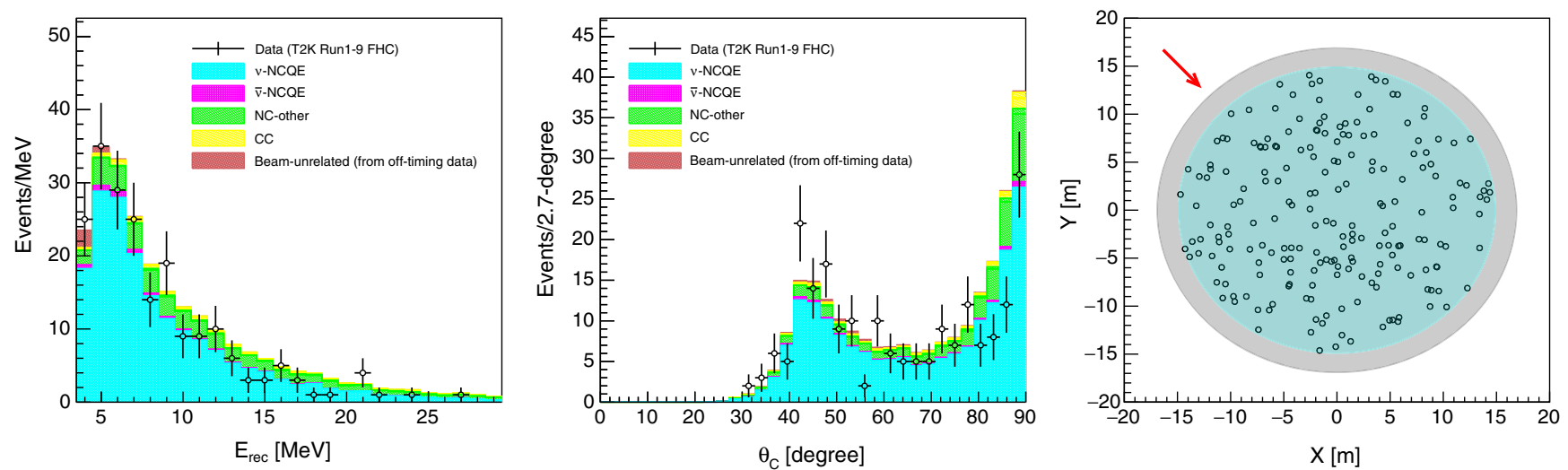

FIG. 7. Distributions of $E_{\mathrm{rec}}$ (left), $\theta_{\mathrm{C}}$ (middle), and vertex (right) from the FHC sample. In the right panel, the red arrow indicates the beam direction and the gray and sky blue regions correspond to the ID and FV, respectively. 

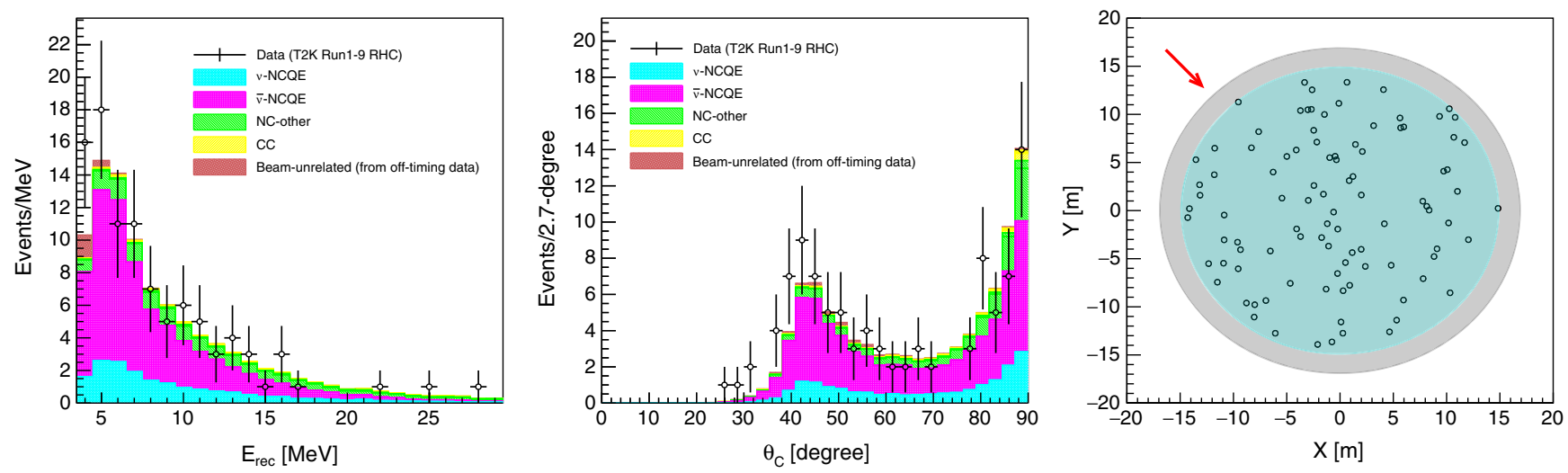

FIG. 8. Distributions of $E_{\text {rec }}$ (left), $\theta_{\mathrm{C}}$ (middle), and vertex (right) from the RHC sample. In the right panel, the red arrow indicates the beam direction and the gray and sky blue regions correspond to the ID and FV, respectively.

(3) The final phase of the event selection is focused on the removal of $\mathrm{CC}$ interaction events. A single charged particle whose momentum is large compared to its mass is likely to have a Cherenkov angle of $\sim 42^{\circ}$ in water. On the other hand if the particle momentum is lower, the reconstructed Cherenkov angle decreases. In this analysis low energy muons from CC interactions and still above Cherenkov threshold distribute around $\theta_{\mathrm{C}}=20^{\circ}-35^{\circ}$, whereas decay-e's have $\theta_{\mathrm{C}} \sim 42^{\circ}$. The contribution of each can be seen in Fig. 5. To reduce these CC events, a linear cut in the reconstructed energy and Cherenkov angle plane is chosen by maximizing the FOM defined in Eq. (3). In the figure the resulting cut is shown with a red line. This is performed separately for the FHC and RHC samples. Using the optimized cut the signal efficiency is $99 \%$ (99\%) while $63 \%$ (58\%) of CC events are removed in FHC (RHC) mode. Some CC-other events still remain after this cut, which could be due to, for example, multiple- $\gamma$ emission via neutron production (as explained later), but this fraction is small with respect to the total number of selected events. Similar population is seen also in the NC-other distribution.

After all cuts, the event selection is more than $80 \%$ efficient for signal events, while reducing background events by more than two orders of magnitude. Figure 6

TABLE I. Number of events after each cut in data and MC. Before the timing cut, only the beam quality and detector condition cuts are applied.

\begin{tabular}{|c|c|c|c|c|c|c|c|}
\hline \multirow[b]{2}{*}{$\mathrm{FHC}$} & \multirow{2}{*}{$\frac{\text { Observation }}{\text { On-timing data }}$} & \multicolumn{6}{|c|}{ Prediction } \\
\hline & & Total & $\nu$-NCQE & $\bar{\nu}$-NCQE & NC-other & $\mathrm{CC}$ & Beam-unrelated \\
\hline Timing cut & 4595 & $\ldots$ & $\ldots$ & $\ldots$ & $\ldots$ & $\ldots$ & 4357.5 \\
\hline Decay-e cut & 4553 & $\ldots$ & $\ldots$ & $\ldots$ & $\ldots$ & $\ldots$ & 4350.8 \\
\hline FV cut & 831 & 896.8 & 190.7 & 5.2 & 52.1 & 24.9 & 623.9 \\
\hline$d w a l l$ cut & 735 & 791.4 & 190.0 & 5.2 & 51.9 & 24.8 & 519.5 \\
\hline effwall cut & 442 & 492.7 & 185.6 & 5.0 & 51.4 & 24.6 & 226.1 \\
\hline ovaQ cut & 220 & 263.9 & 181.0 & 4.9 & 50.2 & 24.1 & 3.7 \\
\hline $\mathrm{CC}$ cut & 204 & 238.4 & 178.6 & 4.8 & 42.5 & 8.9 & 3.6 \\
\hline
\end{tabular}

\begin{tabular}{|c|c|c|c|c|c|c|c|}
\hline \multirow[b]{2}{*}{$\mathrm{RHC}$} & \multirow{2}{*}{$\frac{\text { Observation }}{\text { On-timing data }}$} & \multicolumn{6}{|c|}{ Prediction } \\
\hline & & Total & $\nu$-NCQE & $\bar{\nu}$-NCQE & NC-other & $\mathrm{CC}$ & Beam-unrelated \\
\hline Timing cut & 3626 & $\ldots$ & $\ldots$ & $\ldots$ & $\ldots$ & $\ldots$ & 3746.9 \\
\hline Decay-e cut & 3597 & $\ldots$ & $\ldots$ & $\ldots$ & $\ldots$ & $\ldots$ & 3470.0 \\
\hline $\mathrm{FV}$ cut & 613 & 606.0 & 19.6 & 60.7 & 19.6 & 5.7 & 500.4 \\
\hline$d w a l l$ cut & 535 & 524.1 & 19.5 & 60.5 & 19.5 & 5.7 & 418.9 \\
\hline effwall cut & 282 & 279.4 & 19.1 & 58.7 & 19.3 & 5.6 & 176.7 \\
\hline ovaQ cut & 101 & 101.8 & 18.5 & 57.0 & 18.7 & 5.5 & 2.1 \\
\hline $\mathrm{CC}$ cut & 97 & 94.3 & 17.9 & 56.5 & 15.5 & 2.3 & 2.1 \\
\hline
\end{tabular}


shows a comparison of the number of $\mathrm{MC}$ beam neutrino events against beam-unrelated events both before and after these cuts. The event selection summary for the beam data and MC is shown in Table I. After the event selection, 204 events are observed in the FHC data and 97 events are observed in the RHC data. These are compared with the number of predicted events in Table I. While the FHC sample has a high signal purity, the neutrino component forms nearly $20 \%$ of the RHC sample because of the difference between the neutrino and antineutrino cross sections. Figures 7 and 8 show distributions of the reconstructed energy, Cherenkov angle, and vertex position for the FHC and RHC samples, respectively. The observed $E_{\mathrm{rec}}$ distributions agree well with the predictions in both FHC and RHC modes. A clear contribution from $\sim 6 \mathrm{MeV}$ $\gamma$ rays is observed in both operation modes. In the FHC $\theta_{\mathrm{C}}$ distribution, the data at high angles is below the MC expectation, while no such MC excess is seen in the RHC data. This excess was also observed in the previous T2K measurement [20] although the statistical error was larger. At high angles this distribution is dominated by events with multiple $\gamma$ rays. Such events are caused mainly by fast neutron interactions with nuclei in the water. The excess in FHC may then be attributed to inaccurate modeling of secondary neutron reactions and their subsequent $\gamma$ ray emissions. The fact that the disagreement between observation and prediction is visible in FHC and not in RHC, may be understood by the difference in the out-going nucleon kinematics between neutrino and antineutrino interactions. Helicity conservation in antineutrino interactions produces more forward-going leptons in the final state and consequently lower momentum nucleons. The latter therefore goes on to produce fewer secondary $\gamma$ rays than that from its neutrino interaction counterpart. Comparing the ratio of the single $\gamma$ peak $\left(\sim 42^{\circ}\right)$ to the multiple- $\gamma$ peak $\left(\sim 90^{\circ}\right)$ of the MC in each figure, there are relatively fewer events in the high-angle region of the RHC sample. The vertex positions of selected events in the data are found to be uniform and no bias relative to the beam direction is observed.

\section{UNCERTAINTY ESTIMATES}

Based on the observed number of events in Table I, the associated statistical error is $7.0 \%$ for the FHC sample and $10.2 \%$ for the RHC sample.

Systematic errors from six main sources are considered in the analysis, namely the neutrino flux prediction, the neutrino interaction model, the primary- $\gamma$ and secondary- $\gamma$ emission models, neutrino oscillation parameters, and the detector response. In this analysis, $\mathrm{CC}$ measurement results from the $\mathrm{T} 2 \mathrm{~K}$ near detectors are not used so as to ensure that flux and interaction systematics are treated independently. Only statistical uncertainties are considered for beam-unrelated events, $3.0 \%$ in the FHC sample and $3.9 \%$ in the RHC sample, since they are also part of the observed data and respond to detector uncertainties in the same way. The effect of possible rate fluctuations between

TABLE II. Summary of systematic uncertainties on the observed event rate in percent for each sample component. The fraction of each component, listed as "Event fraction," is also shown in percent. For beam-unrelated events the total error entry represents the statistical uncertainty.

\begin{tabular}{lccccc}
\hline \hline FHC & $\nu$-NCQE & $\bar{\nu}$-NCQE & NC-other & CC & Beam-unrelated \\
\hline Event fraction & 75.0 & 2.0 & 17.8 & 3.7 & 1.5 \\
\hline Neutrino flux & 6.7 & 8.6 & 7.3 & 6.4 & $\ldots$ \\
Neutrino interaction & 3.0 & 3.0 & 8.2 & 16.5 & $\ldots$ \\
Primary- $\gamma$ production & 11.0 & 10.6 & 6.0 & 6.6 & $\ldots$ \\
Secondary- $\gamma$ production & 13.5 & 13.4 & 19.5 & 17.6 & $\ldots .1$ \\
Oscillation parameter & $\ldots$ & $\ldots$ & $\ldots$ & 5.2 & $\ldots$ \\
Detector response & 3.4 & 3.4 & 2.0 & 26.7 & $\ldots$ \\
Total error & 19.2 & 19.7 & 23.3 & $\mathrm{CC}$ & Beam-unrelated \\
\hline \hline RHC & $\nu$-NCQE & $\bar{\nu}$-NCQE & NC-other & 2.5 & 2.1 \\
\hline Event fraction & 19.0 & 59.9 & 16.5 & 6.5 & $\ldots$ \\
\hline Neutrino flux & 7.0 & 6.4 & 7.0 & 38.2 & $\ldots$ \\
Neutrino interaction & 3.0 & 3.0 & 10.8 & 3.5 & $\ldots$ \\
Primary- $\gamma$ production & 12.2 & 11.4 & 19.3 & 21.4 & $\ldots$ \\
Secondary- $\gamma$ production & 13.6 & 13.1 & $\ldots$. & 3.1 & $\ldots$ \\
Oscillation parameter & $\ldots$ & $\ldots .0$ & 5.2 & $\ldots$ \\
Detector response & 3.4 & 3.4 & 23.4 & 44.7 & 3.9 \\
Total error & 20.1 & 19.0 & &
\end{tabular}


the on- and off-timing windows is negligible. Table II summarizes the impact of each of these error categories on the different interaction modes populating the samples. Among them, systematic errors from the secondary- $\gamma$ production model are the leading uncertainties. The error sources are described in detail below.

\section{A. Neutrino flux and interaction model uncertainties}

The impact of neutrino flux and interaction systematic uncertainties in this analysis is estimated by the change in the number of selected events relative to the nominal model under a $1 \sigma$ shift in each error source. The procedure follows previous T2K analyses [20,57,58].

Flux uncertainties are evaluated for each neutrino flavor, horn polarity, and neutrino energy bin. Uncertainties in the hadronic interaction cross section are the dominant contribution to the assigned $6 \%-8 \%$ flux uncertainties. This represents a large improvement over previous T2K analyses, due to improved hadron production and interaction constraints from NA61/SHINE measurements using a replica of the T2K target [38].

The value of the axial-vector mass used to generate quasielastic interactions with its $1 \sigma$ error is $M_{\mathrm{A}}^{\mathrm{QE}}=1.21 \pm$ $0.18 \mathrm{GeV} / \mathrm{c}^{2}$. Similarly the Fermi momentum in oxygen is taken to be $225 \pm 31 \mathrm{MeV} / \mathrm{c}$. Parameters describing contributions from $2 \mathrm{p} 2 \mathrm{~h}$ interactions, resonant pion production, and deep inelastic scattering follow the assignments in previous analyses $[20,57,58]$. These result in uncertainties of $8.2 \%(10.8 \%)$ for the NC-other and $16.5 \%(38.2 \%)$ for $\mathrm{CC}$ interaction backgrounds in the FHC (RHC) measurement. The larger uncertainty in the RHC CC component, as seen in Table II, is attributed to the different effect of $M_{\mathrm{A}}^{\mathrm{QE}}$. Since $\gamma$ rays are emitted isotropically and SK has $4 \pi$ acceptance, the signal efficiencies are unaffected by neutrino interaction model uncertainties.

It should be noted that while $\mathrm{NC}$ inelastic scattering without nucleon emission, $\nu(\bar{\nu})+{ }^{16} \mathrm{O} \rightarrow \nu(\bar{\nu})+{ }^{16} \mathrm{O}^{*}$, should be present in the selected sample, it is not simulated in this analysis. According to Ref. [59], the sum of cross sections leading to ${ }^{15} \mathrm{O}^{*}$ and ${ }^{15} \mathrm{~N}^{*}$ after the ${ }^{16} \mathrm{O}\left(\nu, \nu^{\prime}\right)$ interaction increases from $6.7 \times 10^{-42} \mathrm{~cm}^{2}$ at $E_{\nu}=$ $50 \mathrm{MeV}$ to $481 \times 10^{-42} \mathrm{~cm}^{2}$ at $E_{\nu}=500 \mathrm{MeV}$, while it is almost constant above $\sim 200 \mathrm{MeV}$. By comparing this to the expected NCQE cross section in Ref. [19], it is found that the NCQE process dominates over the NC inelastic process without nucleon knock-out above $E_{\nu} \sim 200 \mathrm{MeV}$. In addition, the former cross section is $\sim 40$ times larger at $500 \mathrm{MeV}$ and is expected to be even larger at higher energies. In the present measurement the signal is predominantly from neutrinos above $E_{\nu} \sim 500 \mathrm{MeV}$. Assuming that the detection efficiency of $\gamma$ rays produced from the deexcitation of nuclei recoiling from the NC inelastic interaction without nucleon emission is comparable to that of NCQE scattering, a 3\% error on the signal channel is assigned conservatively in consideration of the expected interaction cross section differences. Another possible contribution is from NC interactions on hydrogen, $\nu(\bar{\nu})+{ }^{1} \mathrm{H} \rightarrow \nu(\bar{\nu})+{ }^{1} \mathrm{H}$, where the final state protons may produce $\gamma$ rays via reactions with water. However, the contribution from such interactions is expected to be less than $1 \%$ of that from NCQE interactions on oxygen and therefore does not significantly affect the results of the present measurement.

\section{B. Primary- and secondary- $\gamma$ production uncertainties}

Errors on the primary $\gamma$ ray emission come from the uncertainties on the spectroscopic factors. Calculation of the spectroscopic strength for the $\mathrm{p}_{3 / 2}$ state has been found to be consistent with electron scattering data within 5.4\% [19], which leads to an error on the observed event rate at $\mathrm{T} 2 \mathrm{~K}$ of less than $3 \%$. The uncertainty due to the others state [all other states than $\left(\mathrm{p}_{1 / 2}\right)^{-1},\left(\mathrm{p}_{3 / 2}\right)^{-1}$, and $\left.\left(\mathrm{s}_{1 / 2}\right)^{-1}\right]$ being included into the $\left(\mathrm{s}_{1 / 2}\right)^{-1}$ state in the nominal model is estimated by comparison with an extreme case. Since no significant deviation in the predicted $\mathrm{p}_{3 / 2}$ strength has been observed in $\left(e, e^{\prime} p\right)$ and $(p, 2 p)$ experiments [60,61], others cannot behave like the $\left(\mathrm{p}_{3 / 2}\right)^{-1}$ state. In contrast, the possibility that the others state behaves like the ground state, $\left(\mathrm{p}_{1 / 2}\right)^{-1}$, emitting no $\gamma$ rays, is considered, since this would not contradict any existing data. To model this, the others state is included into $\left(\mathrm{p}_{1 / 2}\right)^{-1}$ instead, and the change in the event rate relative to the nominal model is taken as the systematic error. This results in uncertainties in the 6\%-12\% range for the signal and background modes. This extreme case covers the uncertainties of the $\mathrm{p}_{1 / 2}$ and $\mathrm{s}_{1 / 2}$ spectroscopic strengths. The total error on primary- $\gamma$ production is taken to be the sum in quadrature of above two sources.

The secondary- $\gamma$ emission rate is model-dependent and at present there is insufficient data on $\gamma$ ray emission from neutron-oxygen reactions at energies above $20 \mathrm{MeV}$ [62], which are most relevant for the present work, making model selection difficult. Since different models predict different amounts of $\gamma$ ray emission, to reduce the impact of such model dependence, instead the total number of emitted Cherenkov photons from secondary emission processes is considered. First, the probability $\left(P_{\text {selected }}\right)$ of an event being reconstructed in the 3.49-29.49 MeV energy region of this analysis is estimated as a function of the number of emitted Cherenkov photons using MC. The resulting probabilities for FHC and RHC are shown in Fig. 9. The number of emitted Cherenkov photons $\left(N_{\mathrm{C}}\right)$ can be broken down into three parts,

$$
N_{\mathrm{C}} \simeq N_{\mathrm{C}}^{\text {primary- } \nu}+N_{\mathrm{C}}^{\text {secondary- } n}+N_{\mathrm{C}}^{\text {secondary }-p} .
$$

Here $N_{\mathrm{C}}^{\text {primary- } \nu}$ denotes the contribution from the primary $\gamma$ ray emission and $N_{\mathrm{C}}^{\text {secondary }-n}\left(N_{\mathrm{C}}^{\text {secondary }-p}\right)$ is from 


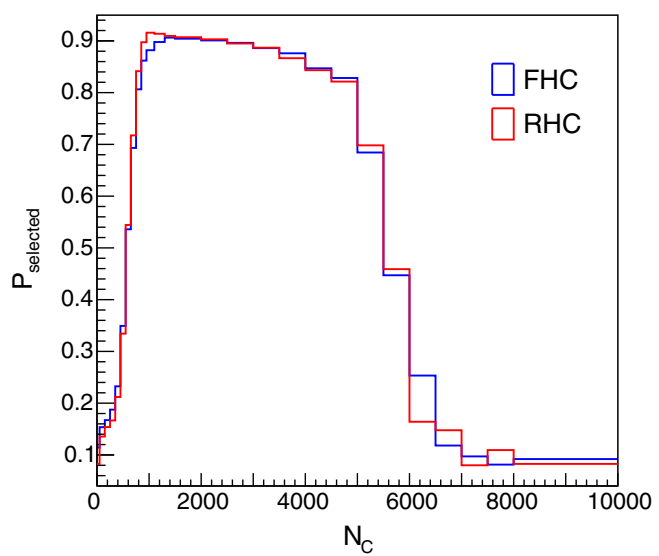

FIG. 9. Probabilities of an event being reconstructed in the energy region of 3.49-29.49 MeV as a function of the number of Cherenkov photons $\left(N_{\mathrm{C}}\right)$ for FHC and RHC.

secondary $\gamma$ rays produced by neutron (proton) interactions in water. The systematic uncertainty used in the analysis is estimated by varying the contributions from these secondary interactions and calculating the change in the selected sample using Fig. 9. The source of uncertainty can be broken down into the initial nucleon-oxygen interaction and the subsequent nuclear de-excitation. In Ref. [63], proton-carbon data were fit to obtain a constraint on the nucleon-nucleus scattering cross section. Their result showed a 30\% difference between the measured and predicted (GCALOR) cross sections. In the present work, the target nucleus is different but the effect is found to be no larger than 5\% in neutrino interaction measurements [58], so a conservative error of $40 \%$ is adopted. In order to estimate the impact of $\gamma$ ray emission from fast neutron reactions on oxygen, the results of a muon-induced spallation study at SK [64] are used. Since the selected sample contains contributions from such neutron interactions, and the measured energy distribution does not differ by more than $50 \%$ from the MC, this number is taken as the error estimate. For the uncertainty propagation the quadratic sum of these two contributions is used and a $\pm 65 \%$ variation is applied to both $N_{\mathrm{C}}^{\text {secondary }-n}$ and $N_{\mathrm{C}}^{\mathrm{secondary}-p}$. The variation producing the largest change in the final sample is used to compute the final error and results in a $\sim 13 \%$ uncertainty for signal and roughly $20 \%$ for the NC-other and CC components. In addition, the impact of uncertainties from the final state interaction model has been evaluated to be as large as $3 \%$. The total uncertainty for each is obtained by summing these two contributions in quadrature.

\section{Oscillation parameter and detector response uncertainties}

Errors on the oscillation parameters, $\theta_{13}, \theta_{23}$, and $\Delta m_{32}^{2}$, are taken from Ref. [23]. Varying each of these, the change in the selected number of $\mathrm{CC}$ events results in 3\%-4\% errors for the FHC and RHC samples.

Errors on each reconstructed parameter used in the event selection, $E_{\text {rec }}$, dwall, effwall, ovaQ, and $\theta_{\mathrm{C}}$ are considered as detector response uncertainties. These have been studied using detector calibrations [54,55], and their effect on the final sample is $1 \%$. Similarly, the gain of the SK PMTs was found to vary over the observation period and its impact is considered as systematic error in this analysis. This gain shift changes the number of PMT hits used to reconstruct energy and produces a $3 \%$ error on the final sample. In total, $3 \%-5 \%$ errors are assigned for each interaction mode.

\section{CROSS SECTION RESULTS}

The number of observed events in the FHC and RHC data ( $D_{\mathrm{obs}}^{\mathrm{FHC}}$ and $D_{\mathrm{obs}}^{\mathrm{RHC}}$, respectively) are expressed as follows:

$$
\begin{aligned}
D_{\mathrm{obs}}^{\text {mode }} & =f_{\nu \text {-NCQE }} M_{\nu \text {-NCQE }}^{\text {mode }}+f_{\bar{\nu} \text { - } \mathrm{NCQE}} M_{\bar{\nu} \text {-NCQE }}^{\text {mode }} \\
& +M_{\mathrm{NC} \text {-other }}^{\text {mode }}+M_{\mathrm{CC}}^{\text {mode }}+D_{\text {beam-unrelated }}^{\text {mode }}
\end{aligned}
$$

where mode $=$ FHC or RHC, $M_{\nu \text {-NCQE }}^{\text {mode }}, M_{\bar{\nu} \text {-NCQE }}^{\text {mode }}, M_{\mathrm{NC} \text {-other }}^{\text {mode }}$, $M_{\mathrm{CC}}^{\text {mode }}$, and $D_{\text {beam-unrelated }}^{\text {mode }}$ represent the expected number of $\nu$-NCQE, $\bar{\nu}$-NCQE, NC-other, CC, and beam-unrelated events, respectively. Here, quantities from the data are written with a capital $D$ while MC predictions are represented with a capital $M$. The factors $f_{\nu \text {-NCQE }}$ and $f_{\bar{\nu} \text {-NCQE }}$ are the measured quantities in the present analysis and serve to scale the NCQE cross section as predicted in the nominal MC model. Based on the observed 204 events in FHC mode and the 97 events in RHC mode the scale factors are calculated to be $f_{\nu-\mathrm{NCQE}}=0.80$ and $f_{\bar{\nu}-\mathrm{NCQE}}=1.11$. Errors on these factors are evaluated using pseudo experiments generated according to random variations of the statistical and systematic uncertainties. Here, statistical uncertainties are considered for $D_{\mathrm{obs}}^{\text {mode }}$ (the effect of the uncertainty from $D_{\text {beam-unrelated }}^{\text {mode }}$ is negligible). Systematic uncertainties are considered for the $M_{\nu \text {-NCQE }}^{\text {mode }}, M_{\bar{\nu} \text {-NCQE }}^{\text {mode }}, M_{\mathrm{NC}-\text { other }}^{\text {mode }}$, and $M_{\mathrm{CC}}^{\text {mode }}$ components. The pseudoexperiments are generated assuming Gaussian distributed error parameters, with means and variances as shown in Tables I and II. Correlations among the flux and cross section parameters are not considered in this analysis. The systematic uncertainty on primary- $\gamma$ production is considered to be fully correlated among the different interaction types and operation modes, and the secondary- $\gamma$ production error is treated in the same way, since the change of the $\gamma$ ray emission rate should be common for the neutrino interaction types and $\mathrm{T} 2 \mathrm{~K}$ operation modes. Note that the primary- and secondary- $\gamma$ production uncertainties are uncorrelated. Distributions of the calculated scale factors for one million pseudo experiments are shown in Figs. 10 and 11. Here the dominant error is the secondary $\gamma$ ray model uncertainty as shown in 

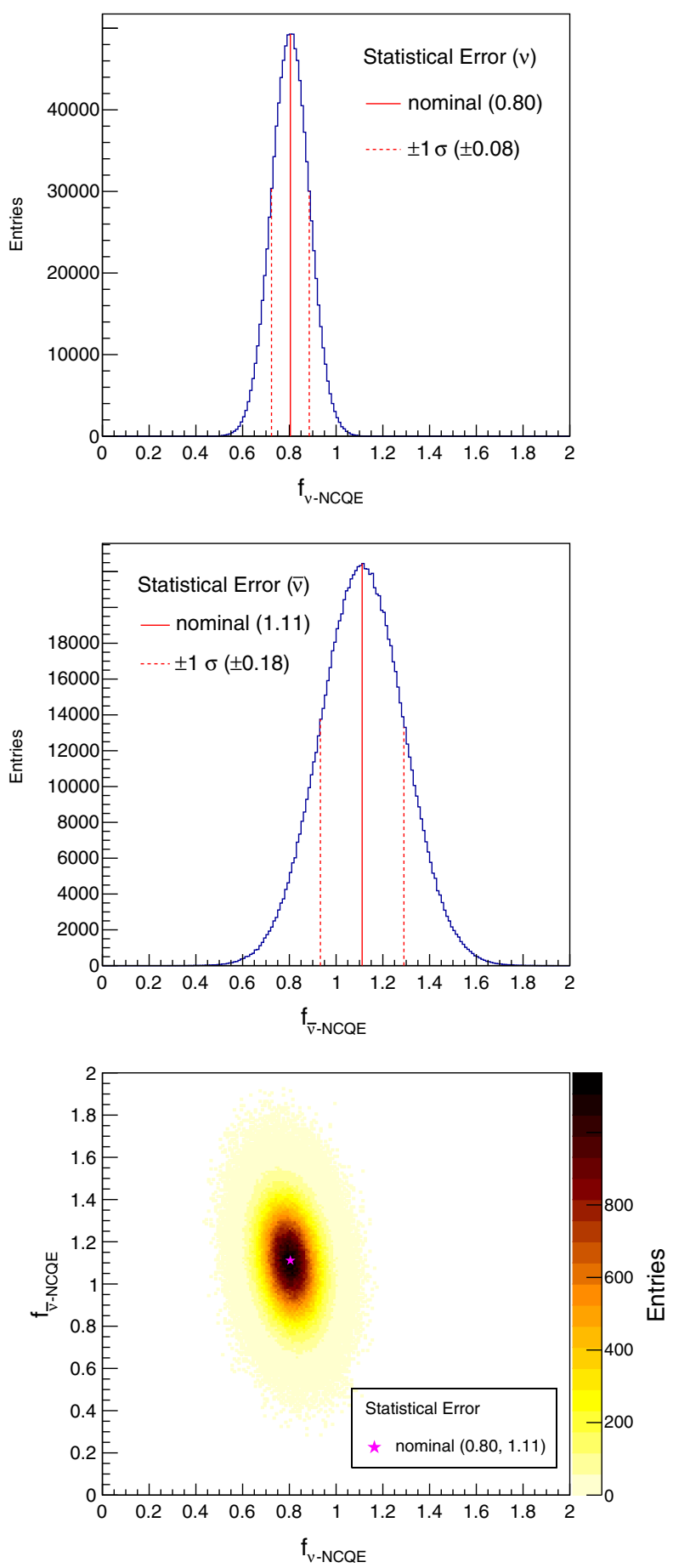

FIG. 10. Results of the pseudoexperiments on the scale factors when the numbers of events are varied based on the statistical uncertainties: $f_{\nu-\mathrm{NCQE}}=0.80 \pm 0.08$ and $f_{\bar{\nu}-\mathrm{NCQE}}=1.11 \pm 0.18$.

Table II. The factors $f_{\nu \text {-NCQE }}$ and $f_{\bar{\nu} \text {-NCQE }}$ have a weak negative correlation for variations of the statistical uncertainty but a strong positive correlation under the influence of systematic uncertainties. In the end, the scale factors are measured as:

$$
f_{\nu-\mathrm{NCQE}}=0.80 \pm 0.08(\text { stat. })_{-0.18}^{+0.24}(\text { syst. })
$$
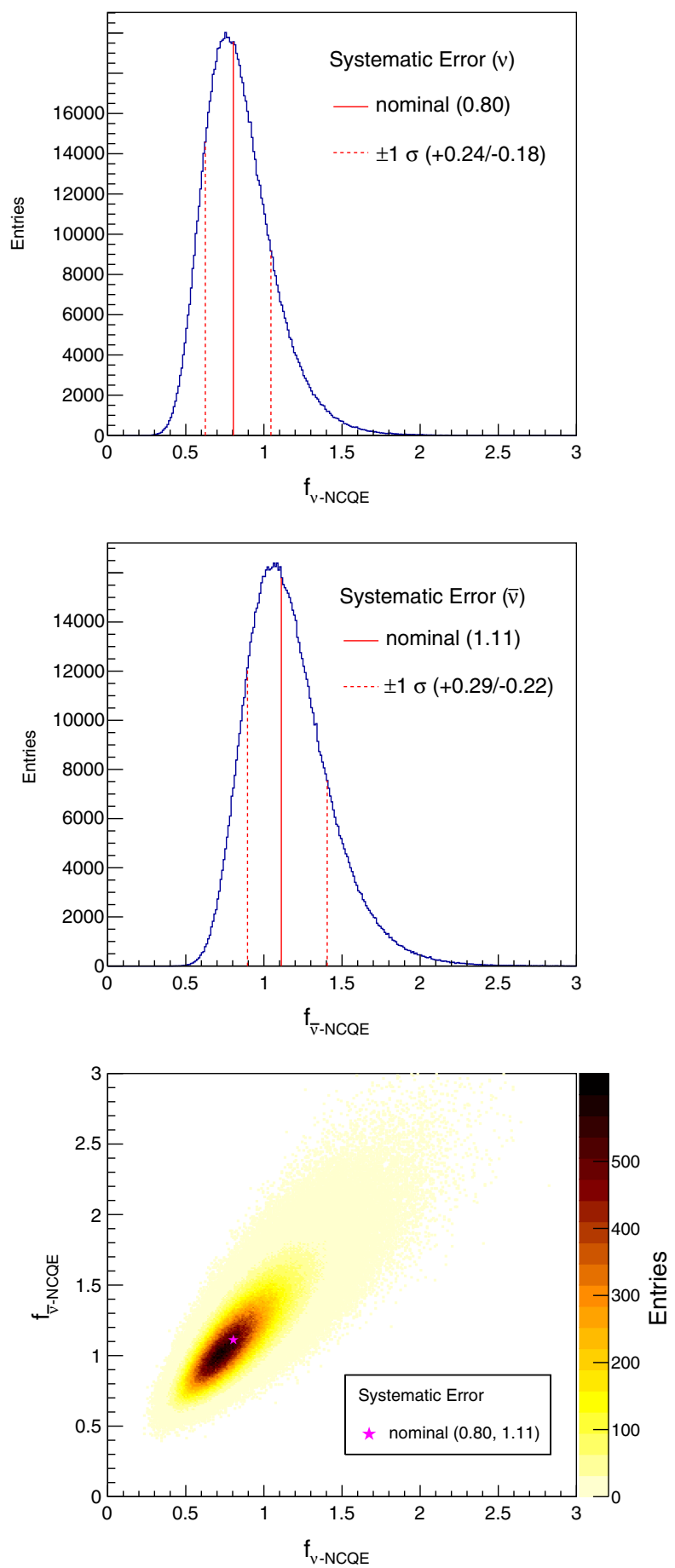

FIG. 11. Results of the pseudoexperiments on the scale factors when the numbers of events are varied based on the systematic uncertainties: $f_{\nu \text {-NCQE }}=0.80_{-0.18}^{+0.24}$ and $f_{\bar{\nu} \text {-NCQE }}=1.11_{-0.22}^{+0.29}$. The dominant uncertainty source is the secondary- $\gamma$ production model as described in the text.

$$
\left.f_{\bar{\nu}-\mathrm{NCQE}}=1.11 \pm 0.18 \text { (stat. }\right)_{-0.22}^{+0.29} \text { (syst.). }
$$

The predictions of flux-averaged cross sections by NEUT for neutrino and antineutrino NCQE interactions on oxygen, $\left\langle\sigma_{\nu \text {-NCQE }}^{\mathrm{NEUT}}\right\rangle$ and $\left\langle\sigma_{\bar{\nu} \text {-NCQE }}^{\mathrm{NEUT}}\right\rangle$, are calculated as: 


$$
\begin{aligned}
\left\langle\sigma_{\nu-\mathrm{NCQE}}^{\mathrm{NEUT}}\right\rangle & =\frac{\sum_{\nu=\nu_{\mu}, \nu_{e}} \int \sigma_{\nu-\mathrm{NCQE}}^{\mathrm{NEUT}}\left(E_{\nu}\right) \phi_{\nu}\left(E_{\nu}\right) d E_{\nu}}{\sum_{\nu=\nu_{\mu}, \nu_{e}} \int \phi_{\nu}\left(E_{\nu}\right) d E_{\nu}} \\
& =2.13 \times 10^{-38} \mathrm{~cm}^{2} / \text { oxygen, }, \\
\left\langle\sigma_{\bar{\nu}-\mathrm{NCQE}}^{\mathrm{NEUT}}\right\rangle & =\frac{\sum_{\nu=\bar{\nu}_{\mu}, \bar{\nu}_{e}} \int \sigma_{\bar{\nu}-\mathrm{NCQE}}^{\mathrm{NEUT}}\left(E_{\nu}\right) \phi_{\nu}\left(E_{\nu}\right) d E_{\nu}}{\sum_{\nu=\bar{\nu}_{\mu}, \bar{\nu}_{e}} \int \phi_{\nu}\left(E_{\nu}\right) d E_{\nu}} \\
& =0.88 \times 10^{-38} \mathrm{~cm}^{2} / \text { oxygen. }
\end{aligned}
$$

The nominal flux, $\phi_{\nu}=\phi_{\nu}^{\mathrm{FHC}}$ is used for neutrinos and $\phi_{\bar{\nu}}=\phi_{\bar{\nu}}^{\mathrm{RHC}}$ is used for antineutrinos in calculations of the flux-averaged NCQE cross sections. Note that summation
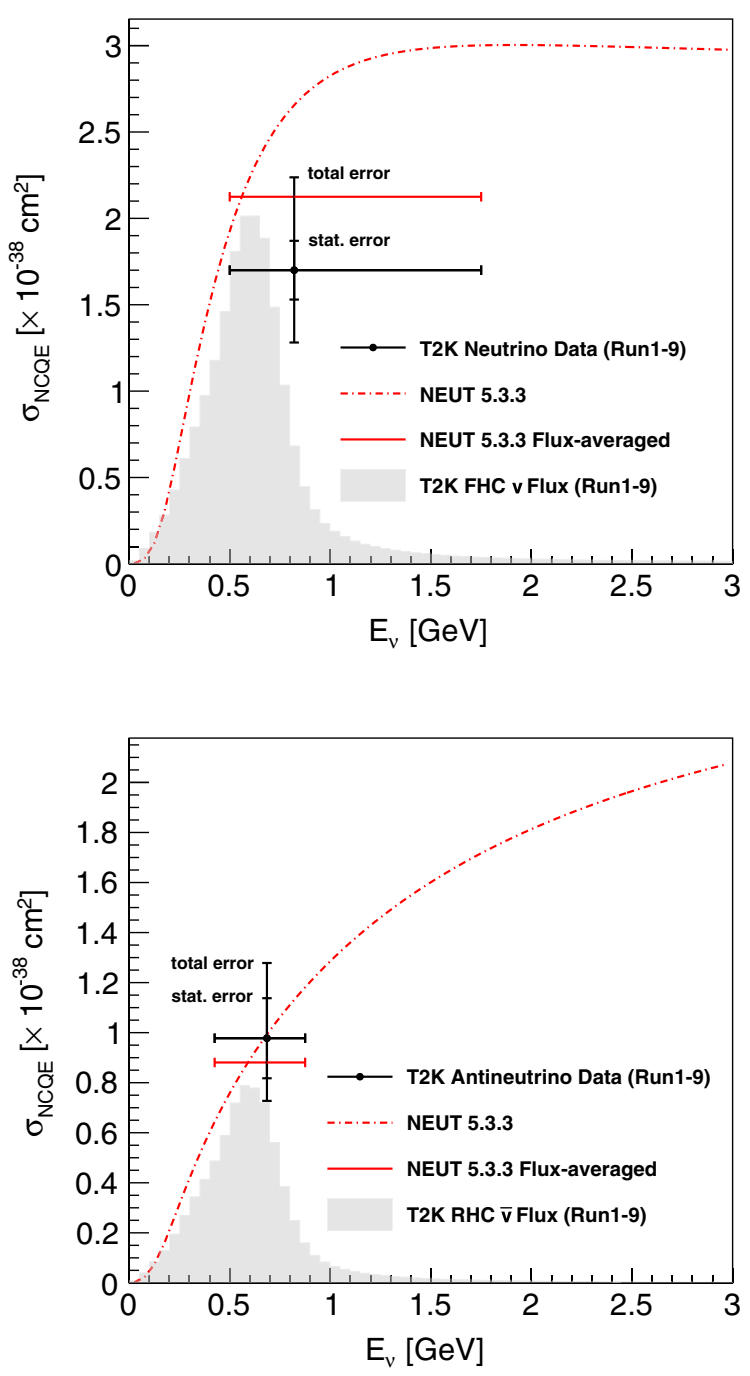

FIG. 12. The measured $\nu$ - (top) and $\bar{\nu}$ - (bottom) ${ }^{16} \mathrm{O}$ NCQE-like cross sections in comparison with the NCQE cross sections predicted by NEUT. The error bars show the statistical error (shorter) and the quadratic sum of statistical and systematic errors (longer). The T2K fluxes for each neutrino beam mode are also shown with an arbitrary normalization. Data points are placed at the mean flux energies, $0.82 \mathrm{GeV}$ for neutrinos and $0.68 \mathrm{GeV}$ for antineutrinos. Horizontal bars represent the upper and lower range of the mean at $1 \sigma$.
TABLE III. Covariance of the neutrino and antineutrino cross sections for the statistical (systematic) error case. The unit of numbers is $\left(10^{-38} \mathrm{~cm}^{2} / \text { oxygen }\right)^{2}$.

\begin{tabular}{lrr}
\hline \hline & $\sigma_{\nu \text {-NCQE }}$ & \multicolumn{1}{c}{$\sigma_{\bar{\nu} \text {-NCQE }}$} \\
\hline$\sigma_{\nu \text {-NCQE }}$ & $0.030(0.227)$ & $-0.005(0.095)$ \\
$\sigma_{\bar{\nu} \text {-NCQE }}$ & $-0.005(0.095)$ & $0.025(0.058)$ \\
\hline \hline
\end{tabular}

is done over muon and electron (anti)neutrinos in Fig. 1, though the actual flux at SK contains tau (anti)neutrinos due to neutrino oscillations. This treatment is justified because the NC cross section is flavor-independent. Here the integrations are conducted up to $10 \mathrm{GeV}$ as higher energies have a negligible impact on the result. The measured flux-averaged NCQE-like cross sections on oxygen nuclei are obtained by multiplying the scale factors to each of Eqs. (8) and (9),

$$
\begin{aligned}
\left\langle\sigma_{\nu \text {-NCQE }}\right\rangle= & f_{\nu \text {-NCQE }} \cdot\left\langle\sigma_{\nu-\mathrm{NCQE}}^{\mathrm{NEUT}}\right\rangle \\
= & \left.1.70 \pm 0.17 \text { (stat. })_{-0.38}^{+0.51} \text { (syst. }\right) \\
& \times 10^{-38} \mathrm{~cm}^{2} / \text { oxygen, } \\
\left\langle\sigma_{\bar{\nu}-\mathrm{NCQE}}\right\rangle= & f_{\bar{\nu}-\mathrm{NCQE}} \cdot\left\langle\sigma_{\bar{\nu} \text {-NCQE }}^{\mathrm{NEUT}}\right\rangle \\
= & \left.0.98 \pm 0.16 \text { (stat. })_{-0.19}^{+0.26} \text { (syst. }\right) \\
& \times 10^{-38} \mathrm{~cm}^{2} / \text { oxygen. }
\end{aligned}
$$

These measurements are shown together with the predictions from NEUT in Fig. 12. The neutrino measurement improves over the previous T2K result with FHC data, $\left\langle\sigma_{\nu \text {-NCQE }}\right\rangle=1.55_{-0.35}^{+0.71}$ (stat. $\oplus$ syst. $) \times 10^{-38} \mathrm{~cm}^{2} /$ oxygen [20]. Covariance matrices of the neutrino and antineutrino flux-averaged NCQE-like cross sections are shown for both variations of the statistical and systematic uncertainties in Table III.

\section{DISCUSSION}

\section{A. NC 2p2h}

Currently, there are no models available in the literature for the NC $2 \mathrm{p} 2 \mathrm{~h}$ interaction, so this channel is not simulated in the present analysis. Since NC 2p2h interactions involve multinucleon knock-out, not only multiple $\gamma$ rays are expected but additional secondary $\gamma$ rays from the recoil nucleons are expected as well. It should be noted that if this process exists then the selection in this analysis likely includes such events. However, if the ratio of the NC $2 \mathrm{p} 2 \mathrm{~h}$ and $\mathrm{QE}$ cross sections is similar to the corresponding $\mathrm{CC}$ ratio, roughly 5\%-10\% [42], the present measurement will not be sensitive to these events.

\section{B. Comparison with model predictions}

The measured NCQE-like cross sections are tied to NEUT as the underlying model for signal and backgrounds. 
It is interesting to compare the current measurements with various theoretical models. Six models from Ref. [65] are used in the comparison: the spectral function (SF); the relativistic mean field (RMF); the superscaling approach (SuSA); the relativistic Green's function with two different potentials (RGF EDAI and RGF Democratic); and the relativistic plane wave impulse approximation (RPWIA) [40,66-69]. The flux-averaged NCQE cross sections for each model are compared in Fig. 13. While the measured result for neutrinos is consistent with all of the models within the $1 \sigma$ error, the SF, RMF, and SuSA models lie outside the $1 \sigma$ region for antineutrinos. However, it is important to note that each model has its uncertainties and none of these models contains the NC $2 \mathrm{p} 2 \mathrm{~h}$ process.

\section{Impact on supernova relic neutrino (SRN) searches}

The present work can be used to estimate NCQE backgrounds from atmospheric neutrinos to SRN searches.
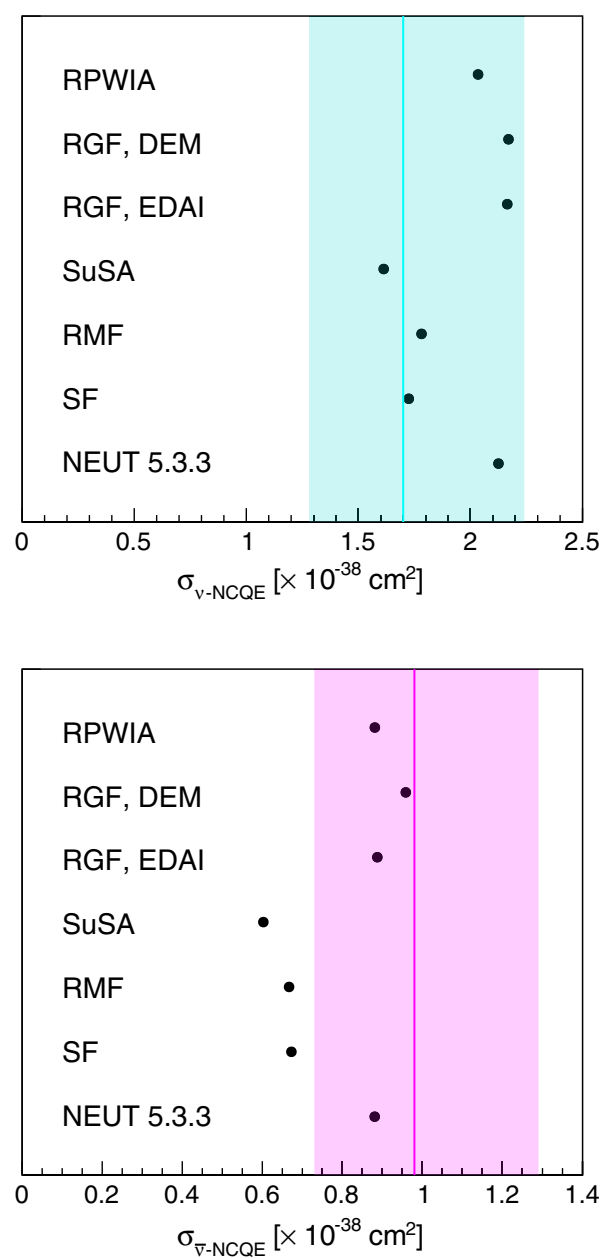

FIG. 13. Comparison of the measured flux-averaged NCQElike cross section to the flux-averaged NCQE cross sections by various models for neutrinos (top) and antineutrinos (bottom). Solid line and shaded area represent the measured mean value and the $1 \sigma$ uncertainty including both statistical and systematic ones, respectively.
Similarly, since $\gamma$ rays from $\mathrm{NC} 2 \mathrm{p} 2 \mathrm{~h}$ interactions are also a background to such searches the inclusive nature of the current measurement may provide useful constraints. Although the cross section results can be used directly, they suffer from large uncertainties from primary- and secondary- $\gamma$ emission models as detailed above. If instead one uses the number of events in the expected SRN signal region, most uncertainties in Table II can be avoided and only errors arising from the difference between the T2K beam and atmospheric neutrino fluxes $(<10 \%)$ and detector response error need to be considered. In the following, the present analysis sample is projected onto the $E_{\text {rec }}-\theta_{\mathrm{C}}$ phase space used in the SK SRN search and divided into four regions: (1) $E_{\mathrm{rec}} \in[3.49,7.49] \mathrm{MeV}$ and $\theta_{\mathrm{C}} \in[38,50]$ degrees, (2) $E_{\mathrm{rec}} \in[7.49,29.49] \mathrm{MeV}$ and $\theta_{\mathrm{C}} \in[38,50]$ degrees (3) $E_{\mathrm{rec}} \in[3.49,7.49] \mathrm{MeV}$ and $\theta_{\mathrm{C}} \in[78,90]$ degrees, and (4) $E_{\text {rec }} \in[7.49,29.49] \mathrm{MeV}$ and $\theta_{\mathrm{C}} \in$ $[78,90]$ degrees. The signal window of the SRN analysis in SK corresponds to region 2 (higher $E_{\text {rec }}$ and lower $\theta_{\mathrm{C}}$ ). Figure 14 gives the $E_{\text {rec }}-\theta_{\mathrm{C}}$ distributions from the FHC
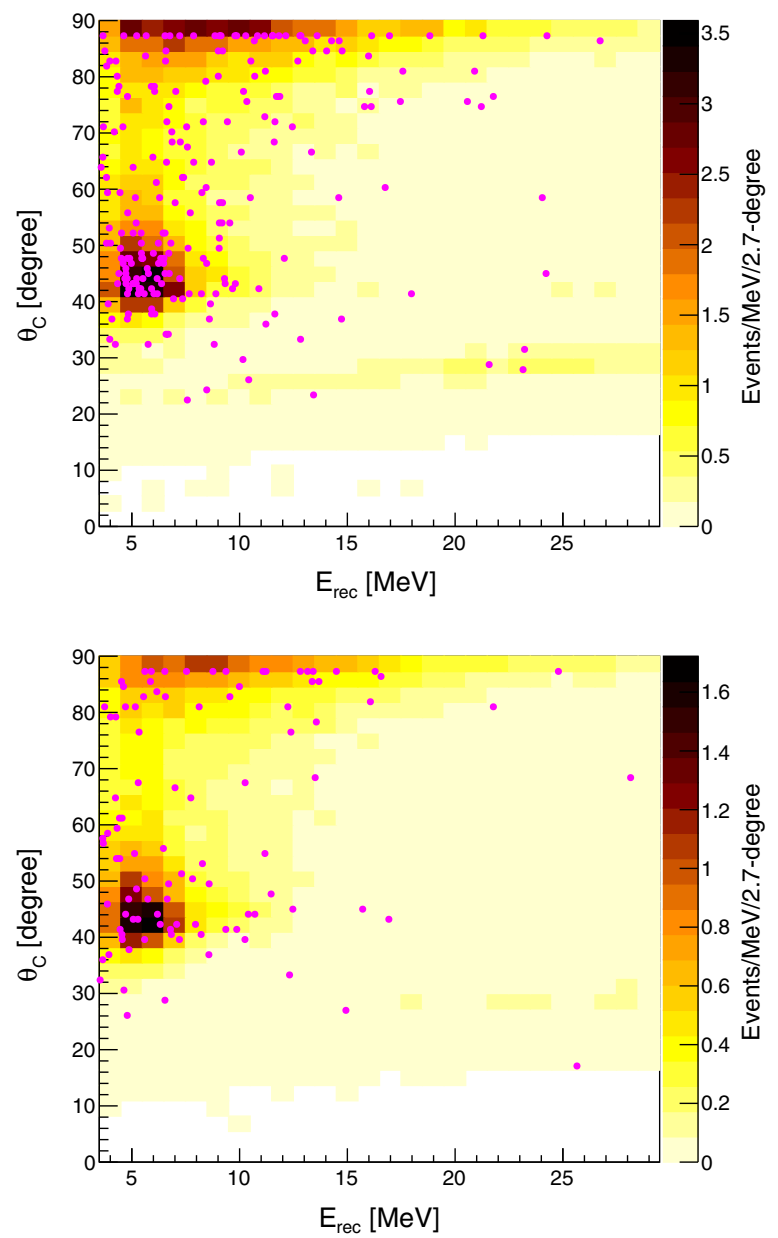

FIG. 14. Two-dimensional $E_{\mathrm{rec}}-\theta_{\mathrm{C}}$ distributions for FHC (top) and RHC (bottom) respectively before the $\mathrm{CC}$ interaction cut and after all of the preceding cuts described in Sec. IV. Magenta dots correspond to the observed data. 

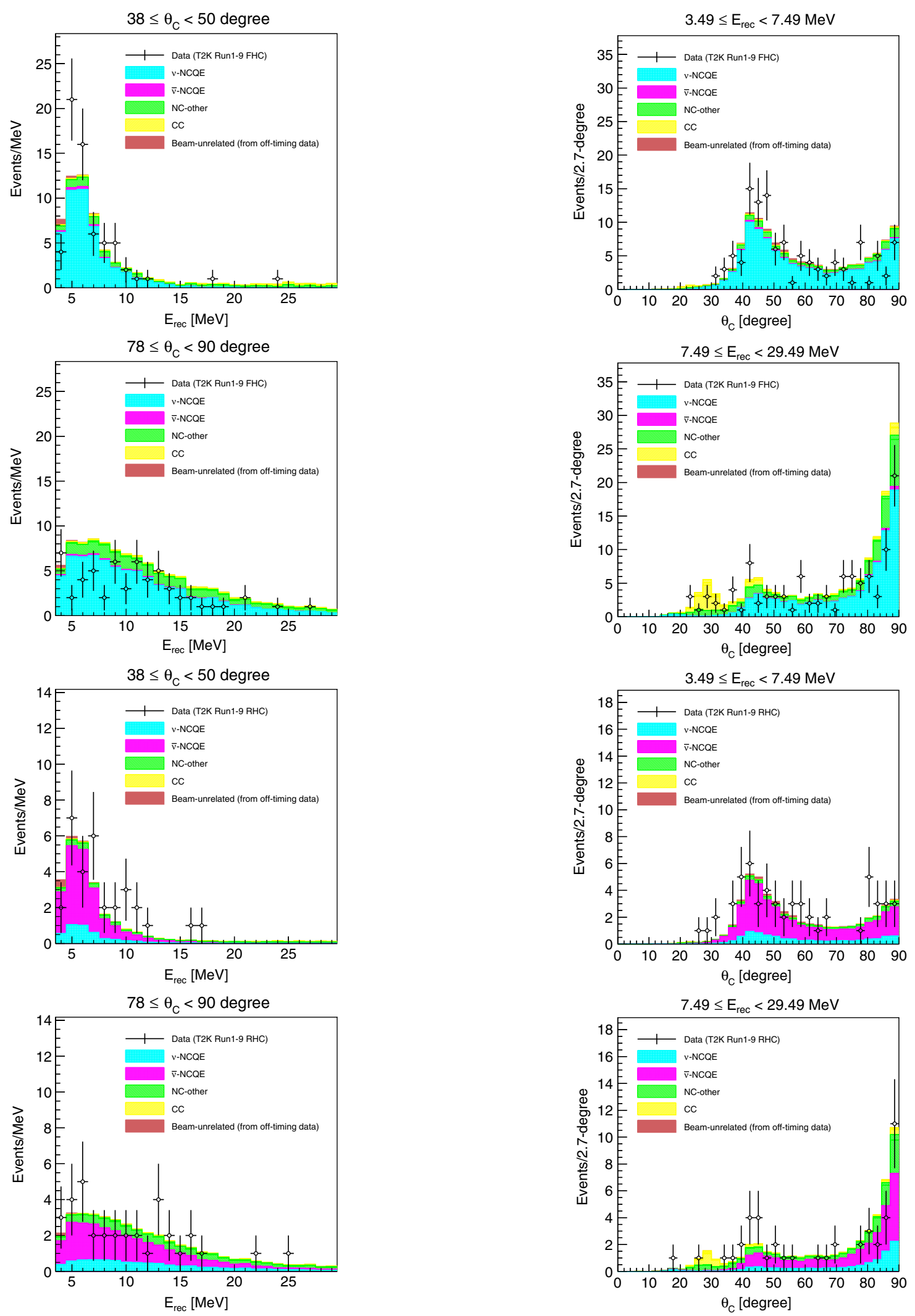

FIG. 15. The $E_{\text {rec }}$ distributions for $\theta_{\mathrm{C}} \in[38,50]$ degrees and $\theta_{\mathrm{C}} \in[78,90]$ degrees before the CC interaction cut and after all of the preceding cuts described in Sec. IV. The top two figures are the FHC results while the bottom two are the RHC results.
FIG. 16. The $\theta_{\mathrm{C}}$ distributions for $E_{\mathrm{rec}} \in[3.49,7.49] \mathrm{MeV}$ and $E_{\text {rec }} \in[7.49,29.49] \mathrm{MeV}$ before the CC interaction cut and after all of the preceding cuts described in Sec. IV. The top two figures are the FHC results while the bottom two are the RHC results. 
TABLE IV. Number of observed and predicted events for each region defined in the text.

\begin{tabular}{lcccc}
\hline \hline FHC & Region 1 & Region 2 & Region 3 & Region 4 \\
\hline Observation & 47 & 16 & 18 & 40 \\
\hline Prediction (total) & 41.1 & 20.4 & 30.8 & 73.8 \\
$\nu$-NCQE & 34.8 & 10.7 & 24.4 & 49.6 \\
$\bar{\nu}$-NCQE & 1.1 & 0.3 & 0.6 & 1.3 \\
NC-other & 3.4 & 5.7 & 4.6 & 19.3 \\
CC & 0.8 & 3.6 & 0.7 & 3.6 \\
Beam-unrelated & 1.0 & 0.1 & 0.5 & 0.0 \\
& & & & \\
\hline \hline RHC & \multirow{2}{*}{ Region 1} & Region 2 & Region 3 & Region 4 \\
\hline Observation & 19 & 12 & 14 & 21 \\
\hline Prediction (total) & 18.6 & 7.3 & 11.9 & 27.0 \\
$\nu$-NCQE & 3.2 & 1.1 & 2.2 & 5.7 \\
$\bar{\nu}$-NCQE & 13.4 & 3.4 & 7.5 & 13.1 \\
NC-other & 1.2 & 2.1 & 1.7 & 7.2 \\
CC & 0.1 & 0.7 & 0.2 & 1.0 \\
Beam-unrelated & 0.7 & 0.0 & 0.3 & 0.0 \\
\hline \hline
\end{tabular}

and RHC data and MC before the CC interaction cut and after all of the preceding cuts described in Sec. IV. Table IV summarizes the number of beam events in each region calculated from Fig. 14. Note that the difference between the observed number of events and predictions in regions 3 and 4 for the FHC sample may be attributed to the inaccuracy of the secondary interaction model as explained in Sec. IV. The $E_{\text {rec }}$ distributions for $\theta_{\mathrm{C}} \in$ $[38,50]$ degrees and $\theta_{\mathrm{C}} \in[78,90]$ degrees for the FHC and RHC samples are given in Fig. 15. Similarly, Fig. 16 shows the $\theta_{\mathrm{C}}$ distributions for $E_{\mathrm{rec}} \in[3.49,7.49] \mathrm{MeV}$ and $E_{\mathrm{rec}} \in[7.49,29.49] \mathrm{MeV}$. Here also the FHC distributions for observation and prediction show discrepancies, which may be attributed to modeling of the secondary- $\gamma$ emission. These distributions can be used to estimate the NCQE background to the SRN search by suitable weighting of the MC to data. Though beyond the scope of the present work, this is expected to significantly improve the current $100 \%$ error on this background used in the SK SRN analysis $[8,9]$.

\section{Future prospects}

At present $\mathrm{T} 2 \mathrm{~K}$ has collected less than half of its expected POT and extensions of the experiment are being considered [70]. The larger statistics of future data sets motivate several possible improvements to the present work. Systematic errors from the secondary- $\gamma$ production model can be reduced by incorporating recent measurements of $\gamma$ ray emission from neutron-oxygen interactions into MC. Measurements using 30, 80, and $250 \mathrm{MeV}$ neutrons have been performed, but only results at $80 \mathrm{MeV}$ are available at present [62]. Furthermore, neutron tagging at SK, particularly the high-efficiency tagging realized in the coming Gd-doped phase of SuperKamiokande (SK-Gd), can be used to study the relationship of neutrons, their transport in water, and the production of secondary $\gamma$ rays. Information on the neutron capture vertex would further constrain the neutron kinetic energy in NCQE interactions by measurement of the neutron flight distance from the primary interaction vertex. Neutron information would also allow for differential cross section measurements using the reconstructed $Q^{2}$ as well as studies of $\Delta s$ if proton and neutron final states can be distinguished. Finally, using the $\sim 8 \mathrm{MeV} \gamma$ cascade following neutron capture on $\mathrm{Gd}$, it may be possible to identify the NCQE interactions resulting in the ground state nucleus by requiring no activity by the primary- $\gamma$.

\section{CONCLUSION}

In this paper, neutrino- and antineutrino-oxygen neutralcurrent quasielasticlike interactions have been measured using nuclear de-excitation $\gamma$ rays at the $\mathrm{T} 2 \mathrm{~K}$ far detector, with data corresponding to $14.94 \times 10^{20} \mathrm{POT}$ in FHC and $16.35 \times 10^{20}$ POT in RHC polarities. Compared to the previous $\mathrm{T} 2 \mathrm{~K}$ study, the present analysis has improved the event simulation and selection criteria, and reduced both systematic and statistical uncertainties. In addition, this work presents the first measurement of antineutrino interactions in this channel to date. The measured flux-averaged NCQE-like cross sections on oxygen nuclei are $\left\langle\sigma_{\nu \text {-NCQE }}\right\rangle=$ $1.70 \pm 0.17$ (stat. $)_{-0.38}^{+0.51}$ (syst.) $\times 10^{-38} \mathrm{~cm}^{2} /$ oxygen for neutrinos at a flux-averaged energy of $0.82 \mathrm{GeV}$ and $\left\langle\sigma_{\bar{\nu} \text {-NCQE }}\right\rangle=0.98 \pm 0.16$ (stat. $)_{-0.19}^{+0.26}$ (syst. $) \times 10^{-38} \mathrm{~cm}^{2} /$ oxygen for antineutrinos at a flux-averaged energy of $0.68 \mathrm{GeV}$. Simultaneously treating both FHC and RHC data has resulted in similar sized errors for both the neutrino and antineutrino measurements. These results were found to be consistent with currently available models within the measurement precisions. In addition, MC and data comparisons in the kinematic regions of interest for SRN searches were performed. These measurements are expected to improve estimates of backgrounds to those searches not only in the present Super-Kamiokande experiment, but also in future water Cherenkov detectors such as SK-Gd and Hyper-Kamiokande. The data related to the results presented in this paper can be found in [71].

\section{ACKNOWLEDGMENTS}

We thank the J-PARC staff for superb accelerator performance. We thank the CERN NA61/SHINE Collaboration for providing valuable particle production data. We acknowledge the support of MEXT, Japan; NSERC (Grant No. SAPPJ-2014-00031), NRC and CFI, Canada; CEA and CNRS/IN2P3, France; DFG, Germany; INFN, Italy; National Science Centre (NCN) and Ministry of Science and Higher Education, Poland; RSF (Grant No. 19-12-00325) and Ministry of Science and Higher 
Education, Russia; MINECO and ERDF funds, Spain; SNSF and State Secretariat for Education, Research and Innovation, Switzerland; STFC, UK; and DOE, USA. We also thank CERN for the UA1/NOMAD magnet, DESY for the HERA-B magnet mover system, NII for SINET4, the WestGrid and SciNet consortia in Compute Canada, and GridPP in the United Kingdom. In addition, participation of individual researchers and institutions has been further supported by funds from ERC (FP7), "la Caixa" Foundation (ID 100010434, fellowship code LCF/BQ/ IN17/11620050), the European Union's Horizon 2020 Research and Innovation programme under the Marie Sklodowska-Curie grant agreement no. 713673 and H2020 Grant No. RISE-GA644294-JENNIFER 2020; JSPS, Japan; Royal Society, UK; and the DOE Early Career program, USA.
[1] L. Alvarez-Ruso, Y. Hayato, and J. Nieves, New J. Phys. 16, 075015 (2014).

[2] L. A. Ahrens et al. (BNL E734 Collaboration), Phys. Rev. D 35, 785 (1987).

[3] A. A. Aguilar-Arevalo et al. (MiniBooNE Collaboration), Phys. Rev. D 82, 092005 (2010).

[4] A. A. Aguilar-Arevalo et al. (MiniBooNE Collaboration), Phys. Rev. D 91, 012004 (2015).

[5] S. Fukuda et al. (Super-Kamiokande Collaboration), Nucl. Instrum. Methods Phys. Res., Sect. A 501, 418 (2003).

[6] J. Beacom and M. Vagins, Phys. Rev. Lett. 93, 171101 (2004).

[7] K. Abe et al. (Hyper-Kamiokande Proto-Collaboration), arXiv:1109.3262.

[8] M. Malek et al. (Super-Kamiokande Collaboration), Phys. Rev. Lett. 90, 061101 (2003).

[9] K. Bays et al. (Super-Kamiokande Collaboration), Phys. Rev. D 85, 052007 (2012).

[10] H. Zhang et al. (Super-Kamiokande Collaboration), Astropart. Phys. 60, 41 (2015).

[11] P. deNiverville, D. McKeen, and A. Ritz, Phys. Rev. D 86, 035022 (2012).

[12] P. deNiverville, C.-Y. Chen, M. Pospelov, and A. Ritz, Phys. Rev. D 95, 035006 (2017).

[13] P. Adamson et al. (MINOS Collaboration), Phys. Rev. Lett. 107, 011802 (2011).

[14] P. Adamson et al. (NOvA Collaboration), Phys. Rev. D 96, 072006 (2017).

[15] K. Abe et al. (T2K Collaboration), Phys. Rev. D 99, 071103 (R) (2019).

[16] V. N. Folomeshkin, S. S. Gershtein, M. Yu. Khlopov, M. Gmitro, R. A. Eramzhyan, and L. A. Tosunjan, Nucl. Phys. A267, 395 (1976).

[17] S. S. Gershtejn et al., Yad. Fiz. 22, 157 (1975).

[18] S. Nussinov and R. Shrock, Phys. Rev. Lett. 86, 2223 (2001).

[19] A. Ankowski, O. Benhar, T. Mori, R. Yamaguchi, and M. Sakuda, Phys. Rev. Lett. 108, 052505 (2012).

[20] K. Abe et al. (T2K Collaboration), Phys. Rev. D 90, 072012 (2014).

[21] L. Wan et al. (Super-Kamiokande Collaboration), Phys. Rev. D 99, 032005 (2019).

[22] K. Abe et al. (T2K Collaboration), Nucl. Instrum. Methods Phys. Res., Sect. A 659, 106 (2011).
[23] K. Abe et al. (T2K Collaboration), Phys. Rev. Lett. 121, 171802 (2018).

[24] A. K. Ichikawa, Nucl. Instrum. Methods Phys. Res., Sect. A 690, 27 (2012).

[25] T. Sekiguchi et al., Nucl. Instrum. Methods Phys. Res., Sect. A 789, 57 (2015).

[26] K. Abe et al., Nucl. Instrum. Methods Phys. Res., Sect. A 694, 211 (2012).

[27] P. A. Amaudruz et al. (T2K ND280 FGD Collaboration), Nucl. Instrum. Methods Phys. Res., Sect. A 696, 1 (2012).

[28] N. Abgrall et al. (T2K ND280 TPC Collaboration), Nucl. Instrum. Methods Phys. Res., Sect. A 637, 25 (2011).

[29] K. Matsuoka et al., Nucl. Instrum. Methods Phys. Res., Sect. A 624, 591 (2010).

[30] K. Suzuki et al., Prog. Theor. Exp. Phys. 2015, 053C01 (2015).

[31] Y. Ashida, M. Friend, A. K. Ichikawa, T. Ishida, H. Kubo, K. G. Nakamura, K. Sakashita, and W. Uno, Prog. Theor. Exp. Phys. 2018, $103 \mathrm{H} 01$ (2018).

[32] G. Battistoni, F. Cerutti, A. Fassò, A. Ferrari, S. Muraro, J. Ranft, S. Roesler, and P. R. Sala, AIP Conf. Proc. 896, 31 (2007).

[33] C. F. Brun, R., and S. Giani, CERN Report No. CERNW5013, 1994.

[34] K. Abe et al. (T2K Collaboration), Phys. Rev. D 87, 012001 (2013).

[35] N. Abgrall et al. (NA61/SHINE Collaboration), Phys. Rev. C 84, 034604 (2011).

[36] N. Abgrall et al. (NA61/SHINE Collaboration), Phys. Rev. C 85, 035210 (2012).

[37] N. Abgrall et al. (NA61/SHINE Collaboration), Eur. Phys. J. C 76, 84 (2016).

[38] N. Abgrall et al. (NA61/SHINE Collaboration), Eur. Phys. J. C 79, 100 (2019).

[39] Y. Hayato, Acta Phys. Pol. B 40, 2477 (2009).

[40] O. Benhar, N. Farina, H. Nakamura, M. Sakuda, and R. Seki, Phys. Rev. D 72, 053005 (2005).

[41] R. A. Smith and E. J. Moniz, Nucl. Phys. 43, 605 (1972).

[42] J. Nieves, I. R. Simo, and M. J. V. Vacas, Phys. Rev. C 83, 045501 (2011).

[43] R. Bradford, A. Bodek, H. Budd, and J. Arrington, Nucl. Phys. B, Proc. Suppl. 159, 127 (2006).

[44] D. Rein and L. M. Sehgal, Ann. Phys. (N.Y.) 133, 79 (1981).

[45] M. Gluck, E. Reya, and A. Vogt, Eur. Phys. J. C 5, 461 (1998). 
[46] A. Bodek and U. K. Yang, AIP Conf. Proc. 670, 110 (2003).

[47] K. Abe et al. (T2K Collaboration), Phys. Rev. D 96, 092006 (2017).

[48] C. Zeitnitz and T. A. Gabriel, Proceedings of the International Conference on Monte Carlo Simulation in High Energy and Nuclear Physics (MC93) (World Scientific, Singapore, 1993), https://www.worldscientific.com/ doi/abs/10.1142/9789814534833\#page=391.

[49] C. Zeitnitz and T. A. Gabriel, Nucl. Instrum. Methods Phys. Res., Sect. A 349, 106 (1994).

[50] https://www.nndc.bnl.gov/exfor/endf00.jsp (ENDF/B Web Page).

[51] M. Smy, Proceedings of the 30th International Cosmic Ray Conference (2008), Vol. 5, pp. 1279, http://inspirehep.net/ record/1371616/files/access.pdf.

[52] K. Abe et al. (Super-Kamiokande Collaboration), Phys. Rev. D 83, 052010 (2011).

[53] K. Abe et al. (Super-Kamiokande Collaboration), Phys. Rev. D 94, 052010 (2016).

[54] M. Nakahata et al. (Super-Kamiokande Collaboration), Nucl. Instrum. Methods Phys. Res., Sect. A 421, 113 (1999).

[55] E. Blaufuss et al. (Super-Kamiokande Collaboration), Nucl. Instrum. Methods Phys. Res., Sect. A 458, 638 (2001).

[56] J. Cravens et al. (Super-Kamiokande Collaboration), Phys. Rev. D 78, 032002 (2008).

[57] K. Abe et al. (T2K Collaboration), Phys. Rev. D 96, 052001 (2017).
[58] K. Abe et al., Prog. Theor. Exp. Phys. 2019, 093 C02 (2019).

[59] E. Kolbe, K. Langanke, and P. Vogel, Phys. Rev. D 66, 013007 (2002).

[60] M. Leuschner et al., Phys. Rev. C 49, 955 (1994).

[61] K. Kobayashi et al., arXiv:nucl-ex/0604006.

[62] Y. Ashida et al., arXiv:1902.08964.

[63] W. Y. Ma, E. S. P. Guerra, M. Yu, A. Fiorentini, and T. Feusels (T2K collaboration), J. Phys. Conf. Ser. 888, 012171 (2017).

[64] Y. Zhang et al. (Super-Kamiokande Collaboration), Phys. Rev. D 93, 012004 (2016).

[65] A. Ankowski, M. B. Barbaro, O. Benhar, J. A. Caballero, C. Giusti, R. González-Jiménez, G. D. Megias, and A. Meucci, Phys. Rev. C 92, 025501 (2015).

[66] C. J. Horowitz and B. D. Serot, Nucl. Phys. A368, 503 (1981).

[67] J. E. Amaro, M. B. Barbaro, J. A. Caballero, and T. W. Donnelly, Phys. Rev. C 73, 035503 (2006).

[68] R. Gonzalez-Jimenez, J. A. Caballero, A. Meucci, C. Giusti, M. B. Barbaro, M. V. Ivanov, and J. M. Udías, Phys. Rev. C 88, 025502 (2013).

[69] A. Meucci and C. Giusti, Phys. Rev. D 89, 057302 (2014).

[70] K. Abe et al. (T2K Collaboration), arXiv:1607.08004.

[71] http://t2k-experiment.org/results/2019-NCQE-nuclear-gamma (T2K Web Page). 